\title{
The Pedestrian Evacuation Analyst-Geographic Information Systems Software for Modeling Hazard Evacuation Potential
}

Chapter 9 of Section C, Geographic Information Systems Tools and Applications Book 11, Collection and Delineation of Spatial Data

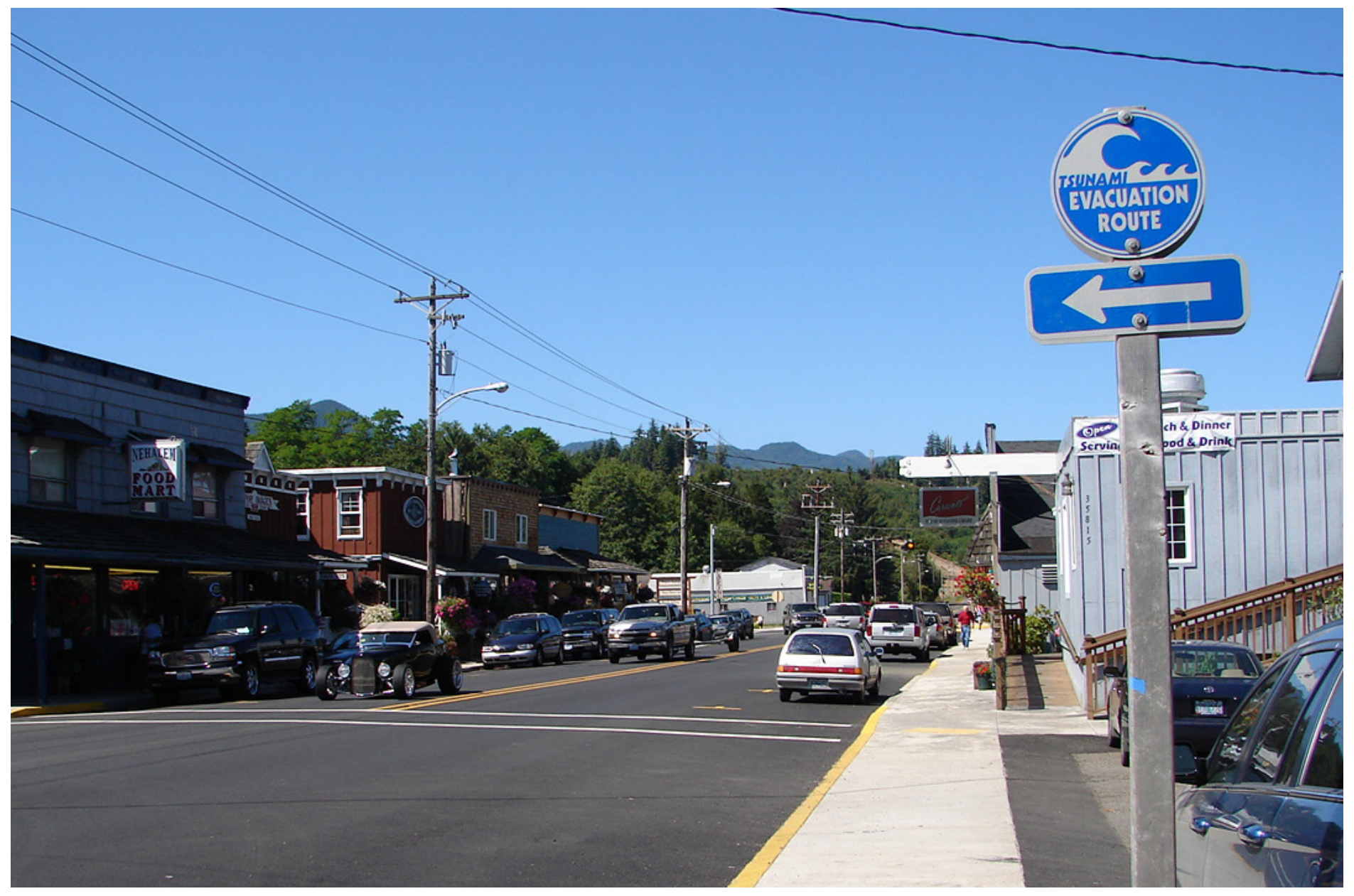

Techniques and Methods 11-C9 
FRONT COVER

Photograph of downtown Nehalem, Oregon, showing the internationally adopted tsunami-evacuation sign (Intergovernmental Oceanographic Commission, 2002). (U.S. Geological Survey photograph by Nathan Wood.) 


\section{The Pedestrian Evacuation Analyst- Geographic Information Systems Software for Modeling Hazard Evacuation Potential}

By Jeanne M. Jones, Peter Ng, and Nathan J. Wood

Chapter 9 of Section C, Geographic Information Systems Tools and Applications

Book 11, Collection and Delineation of Spatial Data

Techniques and Methods 11-C9 


\section{U.S. Department of the Interior SALLY JEWELL, Secretary}

\section{U.S. Geological Survey \\ Suzette M. Kimball, Acting Director}

U.S. Geological Survey, Reston, Virginia: 2014

For product and ordering information: World Wide Web: http://www.usgs.gov/pubprod Telephone: 1-888-ASK-USGS

For more information on the USGS - the Federal source for science about the Earth, its natural and living resources, natural hazards, and the environment: World Wide Web: http://www.usgs.gov

Telephone: 1-888-ASK-USGS

Any use of trade, firm, or product names is for descriptive purposes only and does not imply endorsement by the

U.S. Government.

Although this report is in the public domain, permission must be secured from the individual copyright owners to reproduce any copyrighted material contained within this report.

Suggested citation:

Jones, J.M., Ng, P., Wood, N.J., 2014, The pedestrian evacuation analyst-Geographic information systems software for modeling hazard evacuation potential: U.S. Geological Survey Techniques and Methods, book 11, chap. C9, 25 p., http://dx.doi.org/10.3133/tm11C9. 


\section{Contents}

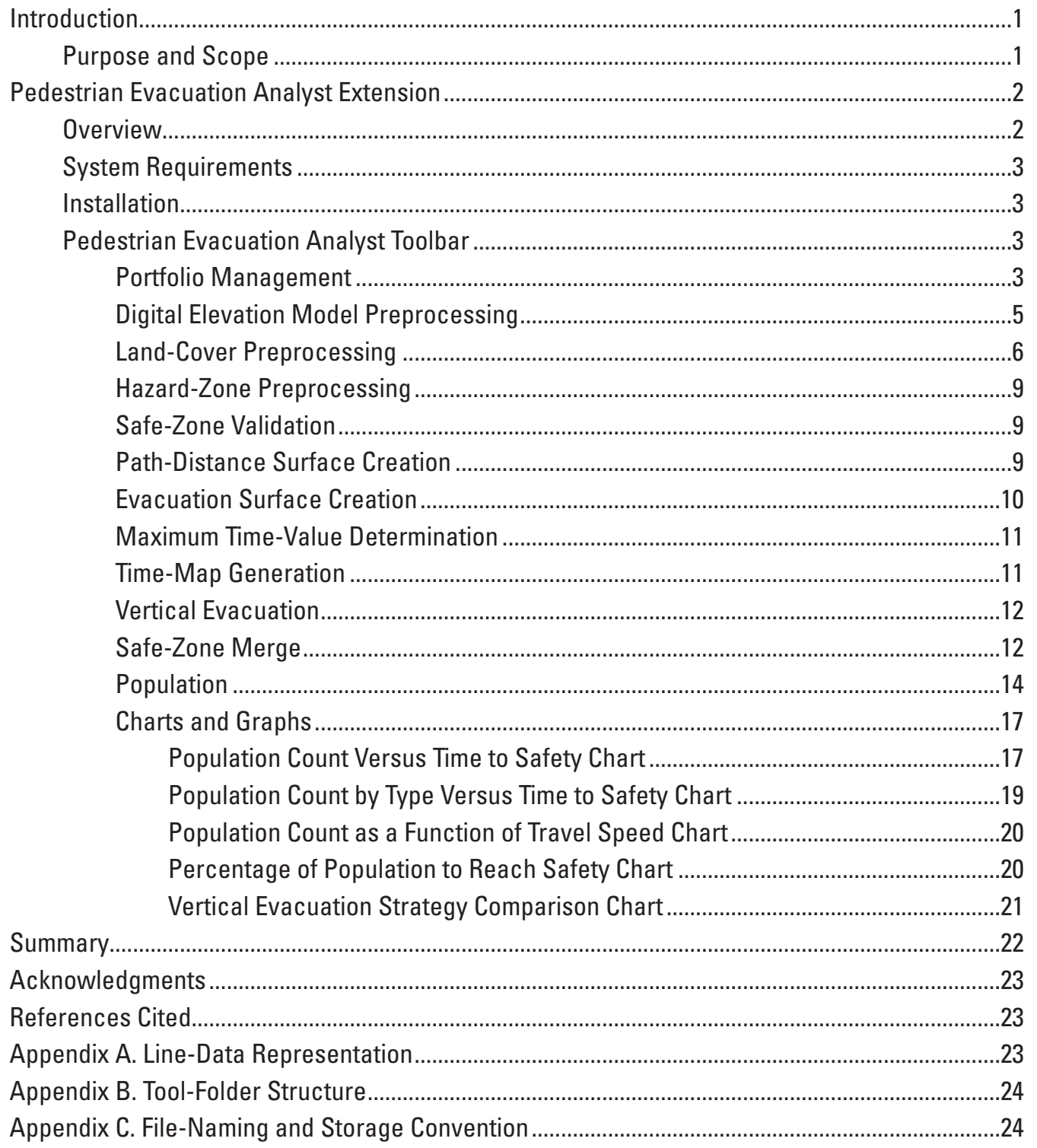




\section{Figures}

1. Tool workflow diagram for the Pedestrian Evacuation Analyst software..................................

2. Diagram of the Pedestrian Evacuation Analyst toolbar ...........................................................

3. Screenshot of the main Portfolio Management window in the Pedestrian Evacuation Analyst. .4

4. Screenshot of the Output tab of Add/Edit Portfolio management window in the Pedestrian Evacuation Analyst. . .4

5. Screenshot of the General tab of Add/Edit Preprocess DEM window in the Pedestrian Evacuation Analyst .6

6. Screenshot of the General tab of Add/Edit LULC window in the Pedestrian Evacuation Analyst .7

7. Screenshot of the Data tab of the Add/Edit LULC Layer window in the Pedestrian Evacuation Analyst..

8. Screenshot of the SCV tab of the Add/Edit LULC Layer window in the Pedestrian Evacuation Analyst...... . .8

9. Screenshot of the LULC Layers tab of Add/Edit LULC window in the Pedestrian Evacuation Analyst.....

10. Screenshots of the hazard zone and study area, tool-derived preliminary safe zone, and valid safe zone after invalid slivers are removed in the Pedestrian Evacuation Analyst

11. Screenshots of Classification windows in the Pedestrian Evacuation Analyst showing histograms for two study areas.

12. Screenshot of Vertical Evacuation tab of the Add/Edit Vertical Evacuation window in the Pedestrian Evacuation Analyst

13. Screenshot from the Pedestrian Evacuation Analyst comparing time maps for baseline processing and vertical evacuation structures for Taholah, Washington.

14. Screenshot of the Population Preprocessing tab of Add/Edit Population window in the Pedestrian Evacuation Analyst

15. Screenshot of the Population Layer tab of Add/Edit Population Layer window in the Pedestrian Evacuation Analyst.

16. Screenshot of the Population Layer Fields tab of Add/Edit Population Layer window in the Pedestrian Evacuation Analyst

17. Screenshot of the Population Table Generation tab of Add/Edit Population window in the Pedestrian Evacuation Analyst

18. Screenshot of the main Charts window in the Pedestrian Evacuation Analyst......................17

19. Screenshot of the Chart Viewer window in the Pedestrian Evacuation Analyst ....................18

20. Screenshot of the Population Count vs. Time to Safety window in the

Pedestrian Evacuation Analyst

21. Screenshot of the Population Count By Type vs Time To Safety window in the Pedestrian Evacuation Analyst

22. Screenshot of a Population Count By Type Vs. Time to Safety chart in the Pedestrian Evacuation Analyst

23. Screenshot of the Data tab in Population Count As a Function of Travel Speed window in the Pedestrian Evacuation Analyst.

24. Screenshot of a Population Count As a Function Of Travel Speed chart results in the Pedestrian Evacuation Analyst...

25. Screenshot of Population Percentage To Reach Safety chart results for Ocean Shores,

Washington, in the Pedestrian Evacuation Analyst. 
26. Screenshot of Vertical Evacuation Strategy Comparison chart results in the

Pedestrian Evacuation Analyst..... . .22

27. Images of line representation in corner-to-corner and staircase formats ............................24

28. Images of algorithm results with corner-to-corner and staircase line representations .......24

\section{Tables}

1. Required input files for various Pedestrian Evacuation Analyst processing steps ...................2

2. Typical land-cover layer ordering for the cost-inverse raster of the

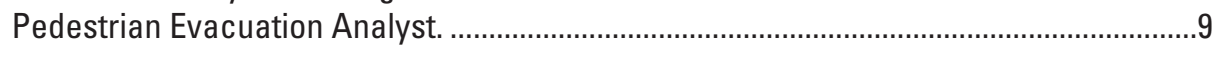

3. Default speed-conservation values and their corresponding land-cover types in the Pedes-

trian Evacuation Analyst...............................................................................................9

4. Travel-speed names and corresponding values in the Pedestrian Evacuation Analyst..........11

5. File-naming and storage convention in the Pedestrian Evacuation Analyst ...........................25 


\title{
The Pedestrian Evacuation Analyst-Geographic Information Systems Software for Modeling Hazard Evacuation Potential
}

\author{
By Jeanne M. Jones, Peter Ng, and Nathan J. Wood
}

\section{Introduction}

Recent disasters such as the 2011 Tohoku, Japan, earthquake and tsunami; the 2013 Colorado floods; and the 2014 Oso, Washington, mudslide have raised awareness of catastrophic, sudden-onset hazards that arrive within minutes of the events that trigger them, such as local earthquakes or landslides. Due to the limited amount of time between generation and arrival of sudden-onset hazards, evacuations are typically self-initiated, on foot, and across the landscape (Wood and Schmidtlein, 2012). Although evacuation to naturally occurring high ground may be feasible in some vulnerable communities, evacuation modeling has demonstrated that other communities may require vertical-evacuation structures within a hazard zone, such as berms or buildings, if at-risk individuals are to survive some types of sudden-onset hazards (Wood and Schmidtlein, 2013).

Researchers use both static least-cost-distance (LCD) and dynamic agent-based models to assess the pedestrian evacuation potential of vulnerable communities. Although both types of models help to understand the evacuation landscape, LCD models provide a more general overview that is independent of population distributions, which may be difficult to quantify given the dynamic spatial and temporal nature of populations (Wood and Schmidtlein, 2012). Recent LCD efforts related to local tsunami threats have focused on an anisotropic (directionally dependent) path distance modeling approach that incorporates travel directionality, multiple travel speed assumptions, and cost surfaces that reflect variations in slope and land cover (Wood and Schmidtlein, 2012, 2013).

The Pedestrian Evacuation Analyst software implements this anisotropic path-distance approach for pedestrian evacuation from sudden-onset hazards, with a particular focus at this time on local tsunami threats. The model estimates evacuation potential based on elevation, direction of movement, land cover, and travel speed and creates a map showing travel times to safety (a time map) throughout a hazard zone. Model results provide a general, static view of the evacuation landscape at different pedestrian travel speeds and can be used to identify areas outside the reach of naturally occurring high ground. In addition, data on the size and location of different populations within the hazard zone can be integrated with travel-time maps to create tables and graphs of at-risk population counts as a function of travel time to safety. As a decision-support tool, the Pedestrian Evacuation Analyst provides the capability to evaluate the effectiveness of various vertical-evacuation structures within a study area, both through time maps of the modeled travel-time landscape with a potential structure in place and through comparisons of population counts within reach of safety.

The Pedestrian Evacuation Analyst is designed for use by researchers examining the pedestrian-evacuation potential of an at-risk community. In communities where modeled evacuation times exceed the event (for example, tsunami wave) arrival time, researchers can use the software with emergency managers to assess the area and population served by potential vertical-evacuation options. By automating and managing the modeling process, the software allows researchers to concentrate efforts on providing crucial and timely information on community vulnerability to sudden-onset hazards.

\section{Purpose and Scope}

The Pedestrian Evacuation Analyst software was developed to aid researchers studying pedestrian, self-initiated evacuation from sudden-onset hazards, such as local tsunamis, debris flows, lahars (volcanic mudflows), and flash floods. Detailed analysis of evacuation potential requires a large amount of high-resolution data and multiple processing steps. The software assists the researcher by managing the data files and providing an orderly path through the processing. Multiple geoprocessing operations are automated into as few steps as possible to free up the researcher's time for further analysis. In addition, graphing tools aid in (1) results visualization by displaying graphs of at-risk populations according to travel times and (2) decision support through comparisons of verticalevacuation options.

The goal is to provide a research tool for exploratory analysis of evacuation potential on the landscape. Results are intended to initiate risk-reduction and preparedness discussions within communities and not serve as definitive statements on the potential mortality from a certain hazard scenario. First, LCD modeling described in this report is based on optimal routes across open landscapes that are determined by mathematical relationships relating slope, land cover, and movement. Actual evacuation pathways taken by at-risk individuals may vary substantially from modeled routes. Second, 
modeling assumes certain travel speeds that may or may not be accurate given the characteristics of the individual or their surroundings (for example, evacuations during a morning rainstorm versus one during a cloudless night sky). Third, modeling travel times to safe zones is based on specific hazard scenarios; therefore, actual safe zones during an event may be different than the model-produced safe zones based on specific scenarios or assumptions.

This paper provides installation instructions for the Pedestrian Evacuation Analyst into Esri's ArcGIS desktop Advanced (ArcInfo) version and describes the steps to run the software and produce evacuation-time maps and population counts. Example maps shown in various figures throughout this report are based on pedestrian-evacuation analyses related to the local tsunami threat in the U.S. Pacific Northwest associated with a Cascadia subduction zone earthquake (Wood and Schmidtlein 2013). Tsunami-wave arrival time in southwest Washington is estimated to be 20-30 minutes after initial ground shaking from the earthquake (Walsh and others, 2000).

\section{Pedestrian Evacuation Analyst Extension}

The overview below of the Pedestrian Evacuation Analyst extension gives a general description of the software. This is followed by a description of software requirements, installation instructions that go through the steps to install it in ArcGIS, and a detailed description of each part of the software and how it is used.

\section{Overview}

The Pedestrian Evacuation Analyst is intended for use by researchers for population-exposure assessments and community outreach to help visualize evacuation scenarios from sudden-onset hazards, such as tsunamis. Specifically, the software was designed to meet the following objectives: (1) automate data preprocessing and evacuation-modeling procedures, (2) calculate travel time to safety from any location in the study area and generate various population counts at each distance, (3) allow travel-speed variations to analyze the effect on travel times and population-exposure numbers, (4) produce a time map for the study area, as well as graphs and charts of population as a function of travel time, and (5) determine the area and population served by potential vertical-evacuation options.

The software installs with a toolbar containing icons for each processing step. The user starts the software by creating or selecting a portfolio as a container for a scenario (step 1; fig. 1). The second step involves preprocessing digital-elevation-model (DEM), land-use/land-cover (LULC), and hazardzone data for use in the evacuation model. The user is able to validate the safe zone developed by the software and, based on the hazard zone, to ensure no errant slivers in the layer exist (for example, due to unintended gaps in hazard polygons). Once all preprocessing is completed, the third step involves several tasks to calculate the path surface, create an evacuation-time surface, and generate a time map. Analysis can stop at this point, or the user can also select the vertical-evacuation (VE) icon to create time maps for any vertical-evacuation option (step 4). Subsequent steps involve generating tables with population counts at different travel times to safety (step 5 ) or creating charts and graphs of the exposed-population data (step 6). The toolbar allows the user to revisit any previous step and rerun with new parameters. Table 1 shows input files required for various processing steps.

\section{Pedestrian Evacuation Analyst Workflow}

1 Create a portfolio for the study area

\begin{tabular}{|c|c|c|}
\hline 2 & Preprocess input data & $\begin{array}{l}\text { Digital elevation model } \\
\text { Land use/land cover } \\
\text { Hazard zone } \\
\text { Safe zone }\end{array}$ \\
\hline 3 & $\begin{array}{l}\text { Create evacuation } \\
\text { surfaces and maps }\end{array}$ & $\begin{array}{l}\text { Calculate path distance } \\
\text { Create evacuation surface } \\
\text { Determine maximum time value } \\
\text { Create time map }\end{array}$ \\
\hline 4 & $\begin{array}{l}\text { Model potential } \\
\text { vertical-evacuation sites }\end{array}$ & $\begin{array}{l}\text { Process vertical-evacuation sites } \\
\text { Merge safe zones }\end{array}$ \\
\hline
\end{tabular}

5 Incorporate population data

6 Develop charts and graphs

Figure 1. Tool workflow diagram for the Pedestrian Evacuation Analyst software.

Table 1. Required input files for various Pedestrian Evacuation Analyst processing steps.

[DEM, digital elevation model; LULC, land use/land cover; VE, vertical evacuation]

\begin{tabular}{|c|c|c|}
\hline Tool name & Input files & File type \\
\hline $\begin{array}{l}\text { DEM } \\
\text { preprocess }\end{array}$ & DEM study-area outline (optional) & Raster Vector \\
\hline $\begin{array}{l}\text { Land-cover } \\
\text { preprocess }\end{array}$ & $\begin{array}{l}\text { Base land cover } \\
\text { Ancillary layers, such as roads, } \\
\text { buildings, water (optional) }\end{array}$ & $\begin{array}{l}\text { Raster or vector } \\
\text { Raster or vector }\end{array}$ \\
\hline $\begin{array}{l}\text { Hazard-zone } \\
\text { preprocess }\end{array}$ & Hazard layer & Vector \\
\hline Path distance & Edited and verified safe zone & Vector \\
\hline $\begin{array}{l}\text { Vertical } \\
\text { evacuation }\end{array}$ & VE locations, polygon & Vector \\
\hline Population & $\begin{array}{l}\text { Multiple population type files, such } \\
\text { as residents, employees, and de- } \\
\text { pendent care (minimum of } 1 \text { file) }\end{array}$ & Vector \\
\hline
\end{tabular}




\section{System Requirements}

- $\quad$ Microsoft Windows 7

- $\quad$.NET Framework 4.0

- $\quad$ Esri ArcGIS for Desktop 10.1, SP1, Advanced (ArcInfo) version with Spatial Analyst extension

The Pedestrian Evacuation Analyst works with ArcGIS versions 9.3 and 10.2 as well, but Esri's update of the path distance tool algorithm in 10.0 introduced bugs that were not corrected until 10.1, SP1. Use of ArcGIS 10.0 is not recommended.

\section{Installation}

Ensure that you are logged into your computer using an account with system administrator privileges. Follow these installation steps to install the tool:

1. Open the Pedestrian Evacuation Analyst installer folder. Double-click the setup.exe file to launch the Pedestrian Evacuation Analyst Setup Wizard page. Click Next.

2. The Select Installation Folder page will prompt for the location where the tool should be installed. Accept the default or modify the location as required. Select the Everyone radio button option to allow everyone to use the tool once it is installed. Click Next.

3. The Confirm Installation page will prompt to begin installing the tool. Click Next. A screen with a status bar will be displayed while the installation is in progress. When installation has completed, the Installation Complete page will be displayed. Click Close.

4. Start ArcMap. From the application menu above the toolbars at the top of the ArcMap window, click Customize > Extensions to display the Extensions window. Select the Evacuation Analyst and Spatial Analyst extensions. Click Close.

5. From the application menu, click Customize $>$ Toolbars and select the Evacuation Analyst. The Pedestrian Evacuation Analyst toolbar will now appear somewhere in the ArcMap application window. Grab the handle (located on the left of the toolbar) and drag the toolbar up into the toolbar menu.

\section{Pedestrian Evacuation Analyst Toolbar}

The Pedestrian Evacuation Analyst toolbar (fig. 2) contains icons for each step in the analysis process. Clicking on an icon opens the main window for each processing step, and clicking the icon again while the window is open will close the window. Initially, the toolbar shows only the Portfolio
Management icon activated and all other icons greyed out, because the user must identify a portfolio (a collection of folder and file names) for use before any other processing steps are valid. Each step is described in the following sections.

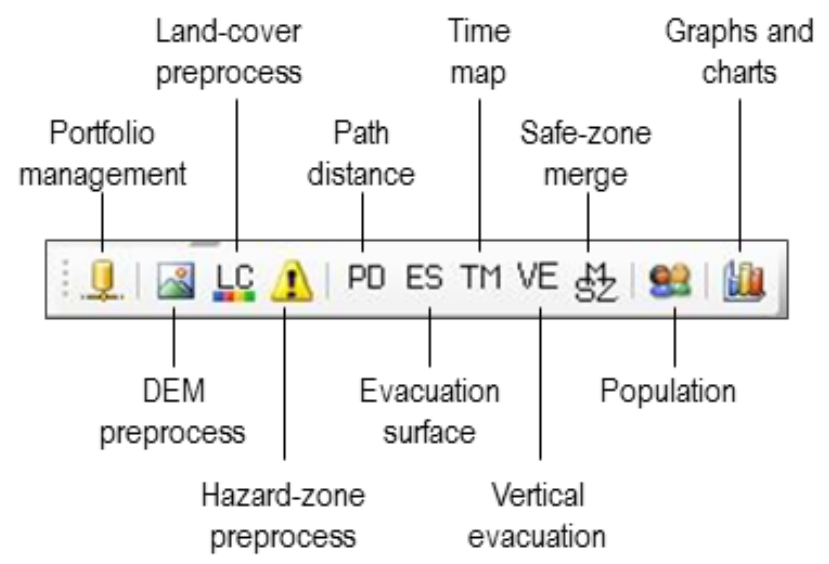

Figure 2. Diagram of the Pedestrian Evacuation Analyst toolbar.

\section{Portfolio Management}

The Pedestrian Evacuation Analyst aids the researcher by simplifying the task of managing the data and results files associated with various processing scenarios. The software uses the portfolio concept to refer to the logical grouping of related files and keeps the names associated with processing scenarios, folder paths, and file paths in a database. A good workflow may begin with creating a portfolio for each study area. Use the portfolio manager to initiate a new portfolio in the database, set a portfolio for use, edit or delete a portfolio that has already been created, or simply verify the settings for a given portfolio.

On first using the Pedestrian Evacuation Analyst, the user will be prompted to create an Evacuation Analyst database in the Portfolio Manager after clicking the Portfolio Management (yellow cylinder) icon (fig. 2). This database will store and manage all the user's transactions performed using the Pedestrian Evacuation Analyst. Click Yes. A folder containing a Pedestrian Evacuation Analyst database is created and stored in the user's system-defined application data folder (that is, "C: $\backslash$ Users $\backslash$ yourname $\backslash$ AppData $\backslash$ Roaming $\backslash$ EvacAnalyst"). The Portfolio Management window (fig. 3) now appears, and the tool is ready for use.

If multiple portfolios (for example, study areas) have been created, the main Portfolio Management window (fig. 3) shows a drop-down list that contains the names of all portfolios in use. Buttons to edit or delete an existing portfolio or to create a new one are located under the name field. The Details button brings up another window that provides more detailed information on the portfolio shown in the bar, including the date created, last date modified, and output locations. The Set button makes the portfolio shown into the active portfolio and activates the rest of the icons in the toolbar. 
To define a new portfolio, the user clicks on the New button, which brings up the Add/Edit Portfolio window. The General tab (not shown) contains a required Name field that is filled in by the user. The Description box provides space to record additional information about the scenario. On the Output tab of this window (fig. 4), the user can use the ellipsis button (...) to navigate to desired folders for workspace, scratch, and results storage. The scratch folder is used for temporary storage of intermediate files during processing. A single scratch folder can be used for all portfolio processing to conserve disk space, and its contents can be deleted when processing is complete. The folder name to use for this portfolio within each work path can be entered in the File/Folder Name box, or just click the AutoFill button and a name will be created from the portfolio name entered. When this portfolio is set as the current portfolio, the folders will be created within the workspace, scratch, and results areas. Note that the contents of the scratch folders, but not the folders themselves, can be deleted by the user between processing steps to free up disk space. The contents of the workspace and results folders are used by different tools in the processing flow and should remain intact. However, when deleting a portfolio, the folders created for that portfolio, as well as all the contents of the folders, are deleted.
Click on the Save button to create the portfolio and dismiss the Add/Edit Portfolio window. The new portfolio will appear in the drop-down list in the Portfolio Management window (fig. 3). Click on the Set button to set the portfolio to the current portfolio. Note that the Portfolio Management window can be opened at any time (by clicking on its icon) and the text at the bottom of the window identifies the currently active portfolio. To activate a different portfolio, select the portfolio from the drop-down list and click Set. Once a portfolio is set, the window can be dismissed by clicking the " $x$ " in the upper right-hand corner or by clicking on the icon again.

The Evacuation Analyst user interface communicates with the geoprocessing tools through text files in XML (extensible markup language) format. These files contain the processing parameter values and input/output file names used in each processing step. The XML files are stored in the results folders of each portfolio (found in the Add/Edit Portfolio window) and can be copied and saved by the user if a written record of processing is desired. In addition, when each time map is created (in subsequent steps) the complete XML file for that time map is written to the results folder. These files contain metadata such as projection and cell size, as well as the contents of all description fields and all input parameters used to create the time map. For users wishing to create formal

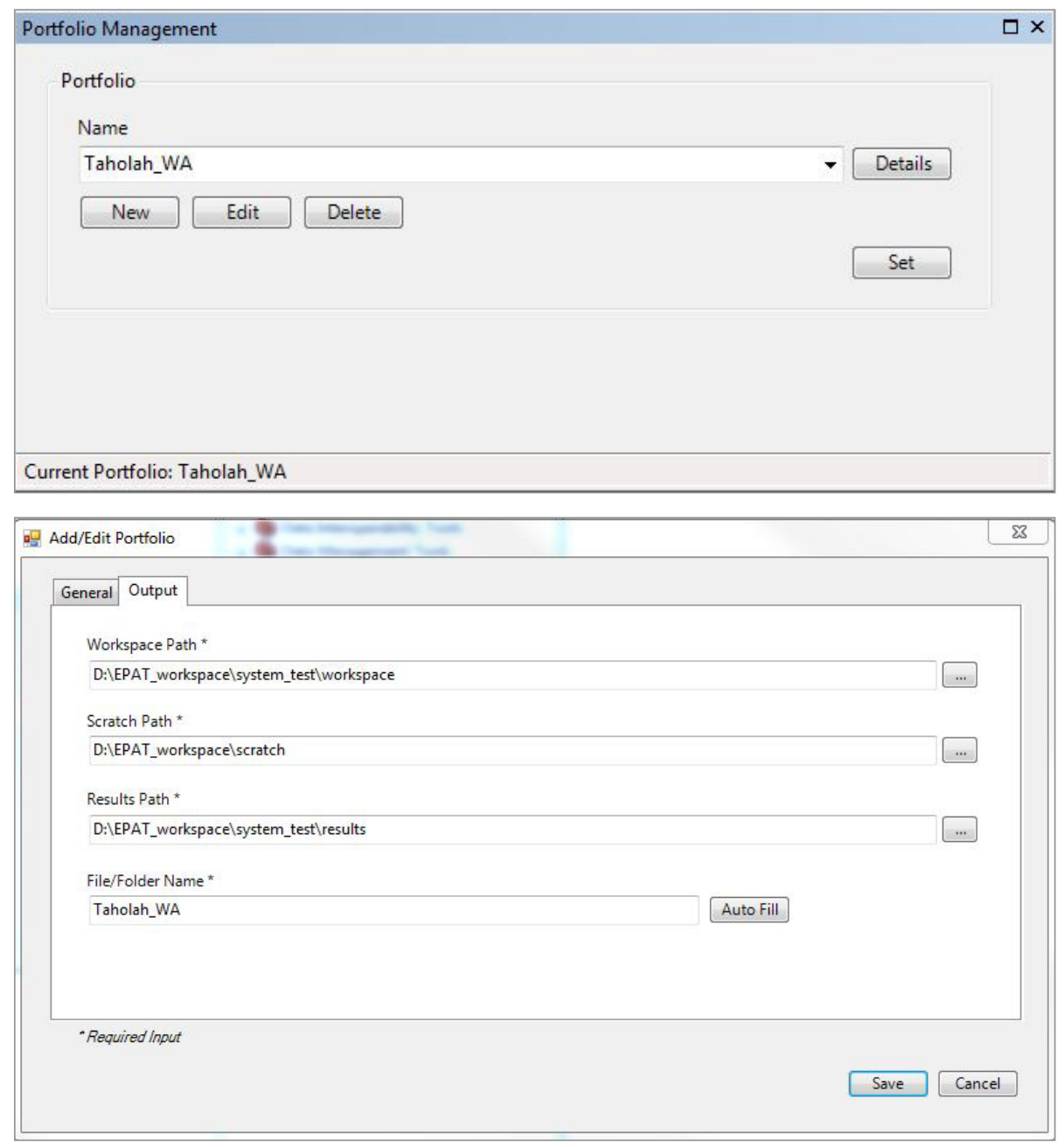

Figure 3. Screenshot of the main Portfolio Management window in the Pedestrian Evacuation Analyst.

Figure 4. Screenshot of the Output tab of Add/Edit Portfolio management window in the Pedestrian Evacuation Analyst. 
metadata, this information is available for use with metadata creation tools (Federal Geographic Data Committee, 2014).

\section{Digital Elevation Model Preprocessing}

Preparing the DEM for processing is the first of the data preprocessing steps. The DEM preprocessing identifies the projection and the study area for the scenario. The user has the option of entering both DEM raster and vector study-area files or only a DEM raster file. If just the DEM is entered, its projection becomes the required projection for the scenario, and its outline is extracted to define the scenario study area. If a separate study-area shapefile is provided initially, then this study-area projection becomes the required projection (the DEM projection must match or it will be rejected), and the DEM is clipped to this study area. The Pedestrian Evacuation Analyst requires that all input files be in the same projection to allow the user to select the proper data transformation for maximum accuracy when converting files from different projections. This step also defines the analysis cell size (in the linear units of the file's projection) for the scenario, and the input DEM will be sampled or aggregated to match the analysis cell size.

The resolution of the input data will have a large impact on the quality of the results. High-resolution elevation data (Wood and Schmidtlein, 2012), detailed and accurate landcover and water data, and up-to-date roads information will produce a more realistic map for travel time to safety than coarser and more generalized data. In particular, coarser resolution elevation was found to underestimate travel times across the hazard zone in a model sensitivity analysis (Wood and Schmidtlein, 2012).

Software execution time increases in proportion to the size of the study area and is also influenced by the amount of available memory and computational speed. Better performance can be obtained by minimizing the size of the study area. If possible, break up a large study area along natural boundaries and mosaic/merge the results together. A natural boundary for modeling would be an area that the algorithm does not need to travel to in order to find the shortest path to a safe haven. For example, a high bluff directly at the ocean's edge works well, as would a long waterway without any connecting bridges. After dividing up a study area, examine the resulting time maps for any edge effects signaling that a path to safety may have been cut off. These edge effects show up as high travel times at the edge of the study area boundary, when a path to a safe haven outside the boundary has been cut off. The following steps describe DEM preprocessing:

1. Click on the DEM Preprocess (mountains with sun image) icon (fig. 2) to open the Add/Edit Preprocess $D E M$ window (click on the icon, fig. 2), and then click on the New button.

2. In the name field of the Add/Edit Preprocess DEM window on the General tab (fig. 5), click on the Autofill button to fill in the name (or type in a different name). This assigns a name to the preprocessed DEM and study area and the user can refer to the files by this name in other processing steps.

3. Fill in the description field to record more detailed information about the processing, if desired.

4. Enter the desired cell size for processing into the Analysis Cell Size field, in the linear units of the study area's projection. If the cell size entered is not the same as the cell size of the input DEM, the raster will be sampled or aggregated to match the given value. For example, if the input DEM has a cell size of 1 meter and a 5 is entered into the Analysis Cell Size field in the Add/Edit Preprocess DEM window (fig. 5), the DEM will be aggregated to a cell size of 5 for processing. Because the input DEM cell size was in meters, the value entered into the Analysis Cell Size field is also interpreted as having linear units of meters.

5. On the DEM tab of the window, click on the Source DEM Raster ellipsis (...) button to navigate to the input, or source, DEM raster. The ellipsis button opens a browser window to search for an input source.

6. The name and location of the preprocessed DEM, called the target DEM raster, are automatically entered in the Target DEM Raster field below. By default, this file will be created in a subfolder of the workspace identified for this portfolio. If desired, the user can navigate to another location for the target file and/or modify the file's name. If the name is modified, be sure to follow ArcGIS's guidelines for GRID file naming by using only letters, numbers or underscore ( $\_$) characters, beginning with a letter, and using only a maximum of 13 characters.

7. On the Study Area tab of the window, navigate to the location of a polygon shapefile containing the study area outline (in the correct projection). If this field is left blank, the tool will create a polygon study-area shapefile from the DEM outline.

8. The file names and locations are automatically filled in for each target file. At the end of DEM preprocessing, the tool stores the study-area shapefile and corresponding study-area raster file in the subfolder of the workspace.

\section{Click the Run button.}

A progress bar will appear while the tool is running. Once the DEM preprocessing has completed, a status window will appear and the target DEM and study-area vector are loaded into ArcMap's table of contents. For information on files and settings used for a processed DEM, open the Preprocess $D E M$ window and click on the Details button after the DEM 


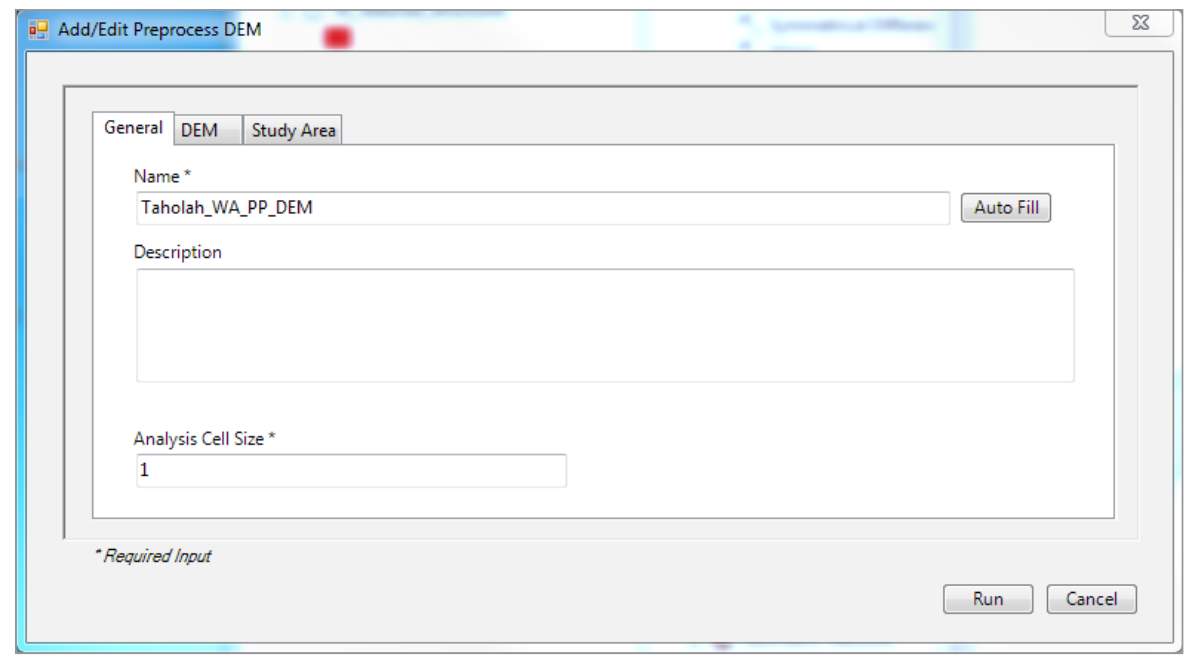

Figure 5. Screenshot of the Genera/ tab of Add/Edit Preprocess DEM window in the Pedestrian Evacuation Analyst.

portfolio name. In the window that opens, information such as date created or modified, projection, cell size, and source and target filenames are displayed for reference.

Note that most tool windows have a Cancel button as a way to exit from the current window without making any changes. The Cancel button closes the current window without updating the database with any new parameters or starting any processing. This button does not halt geoprocessing operations once the Run button has been clicked. Once a geoprocessing operation such as executing path distance has begun, there is no procedure for interrupting or aborting this processing other than forcing ArcMap to quit through the Windows Task Manager.

\section{Land-Cover Preprocessing}

Land-cover data preprocessing combines all inputs related to land cover into a single layer and uses this to create the cost-inverse raster (Wood and Schmidtlein, 2012). A base land-cover layer is the only required file, but additional ancillary files such as roads, water bodies, fences, and buildings can also be added, and these will be overlaid on the base landcover layer according to the order in which they appear in the input list. See table 2 for a typical ordering of land-cover layers. If a land-cover layer contains multiple types (for example, heavy and light brush, wetlands, and beaches), then the layer must have a numeric attribute that describes each land-cover type as a unique integer (for example, "1" for wetland and "2" for light brush).

When entering each land-use/land-cover (LULC) layer, the user sets each unique land-cover type to a specific speedconservation value (SCV) or enters a custom value (table 3). Speed-conservation values represent the fraction of a maximum speed that could be achieved across the given land-cover type (Wood and Schmidtlein, 2012, Soule and Goldman, 1972). Values fall on a spectrum from zero (meaning travel is not possible) to 1.0 (meaning pedestrian evacuation speed is 100 percent of the base travel rate). In addition, this step also performs the basic preprocessing of screening for correct projection, clipping to the study area, and adjusting cell size if necessary. The quality of the cost inverse raster is dependent on the careful selection of input layers, and all input data layers should have a thorough quality-control screening before use in the tool. See also appendix A. The following steps describe land-cover preprocessing:

1. Click on the land-cover preprocess (LC) icon (fig. 2) to open the Preprocess LULC window.

2. To create a new land cover layer, click on the New button.

3. Use the AutoFill button in the Add/Edit LULC window (fig. 6) to get a default name for the preprocessing or enter a name of choice.

4. In the Study Area field, use the drop-down list to select a preprocessed study area generated from the Preprocess DEM step.

5. The name and file path of the resultant (target) cost inverse raster is filled in with default values, and this can be modified if desired.

6. Click on the LULC Layers tab to begin entering landcover layers. Each land-cover file can now be added individually by clicking the $A d d$ button, which brings up the Add/Edit LULC Layer window (fig. 7) to define each layer.

7. Set the Data Type button to the spatial data type of the land cover file currently being entered. The data type can be either raster or vector. See table 2 for a typical ordering of land-cover layers.

8. Use the ellipsis (...) button in the Location field to navigate to the input land-cover source.

9. Set the Layer Type field to indicate whether the landcover layer will serve as either a base layer or an ancillary layer. Land-cover preprocessing consists 
of one base layer and as many ancillary layers as desired.

10. To define the SCVs for the base layer, click on the $S C V$ tab (fig. 8) to define SCV values for each landcover type. The LULC Field Name drop-down list will only show fields with numeric values. Select the numeric field that identifies the land-cover classes and click the Load SCVS button in the lower right corner of the window. This will load all the unique values in the selected field of the attribute table into the LULC Value column of the table. Next to each value is a drop-down list containing a set of SCVs representing the fraction of a maximum speed that could be achieved across the given land-cover type. Default values for various land-cover types are defined in table 2 and are based on terrain energy coefficients discussed in Soule and Goldman (1972). Select the appropriate value for the land-cover type or enter a custom value. When all land-cover values have been assigned an SCV value, click the $O K$ button.

11. To define the SCV values for the ancillary layers, use either the $S C V$ tab or the Ancillary Layer shortcut section on the Data tab. If the ancillary layer contains only one LULC type, use the Ancillary Layer shortcut (described next). If it contains multiple land-cover types, use the $S C V$ tab as described in the previous step.

12. For the Ancillary Layer shortcut, use the drop-down list in the LULC Type field to select the type of land cover in the layer being entered (fig. 7). If none of the choices matches, choose the "Other" category and type in a name for the land-cover type in the Description field. If one of the standard types of land cover is chosen, the Default SCV Value field will be filled with the corresponding $\mathrm{SCV}$ value for that land cover (table 3). This value can be modified if desired. If the "Other" category is selected, type in an appropriate $\mathrm{SCV}$ value. Click the $O K$ button.

13. Once all the layers have been added to the $L U L C$ Layers table (fig. 9), make sure that only one layer has a check mark in the Is Base column. The base layer should be the first layer in the list. If not, use the $U p$ or Down buttons to change the order of the layers in the list. Layers will be merged in the list order from top to bottom, so layers such as water bodies should be merged before a roads layer to keep any bridges in the roads layer from being overlaid by a polygon in the water layer. If any changes need to be made or any layers removed, use the Edit and Delete buttons.

14. Click on the Run button to start the preprocessing.

A progress bar will appear during tool execution, and the status window will be displayed when the LULC preprocessing is complete and the cost-inverse raster will be loaded into ArcMap's table of contents. The newly created preprocessed land cover will appear in the Name list in the Preprocess LULC main window. Land cover processing may take several minutes to run, depending on the analysis cell size, the study area size, and the number of ancillary layers to merge with the base layer. The values in the final cost-inverse raster are the inverted SCVs, with a minimum value of 1.0 and a maximum value of the inverse of the smallest nonzero SCV value entered.

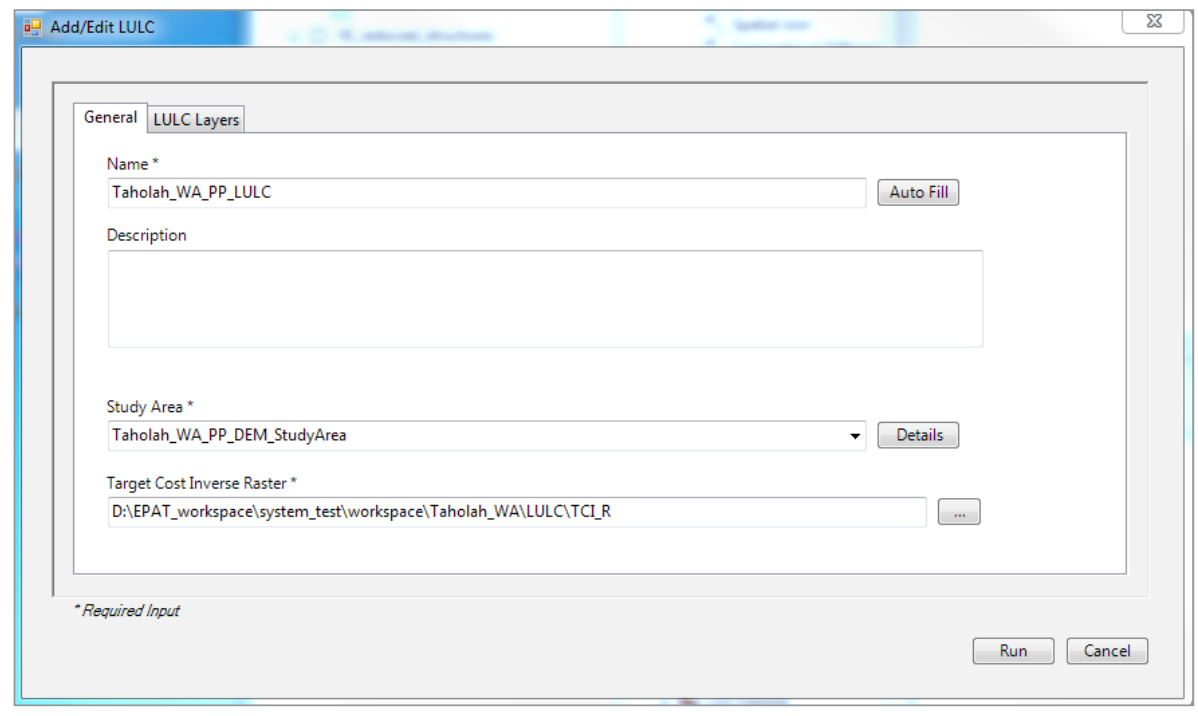

Figure 6. Screenshot of the General tab of $A d d / E d i t ~ L U L C$ window in the Pedestrian Evacuation Analyst. 

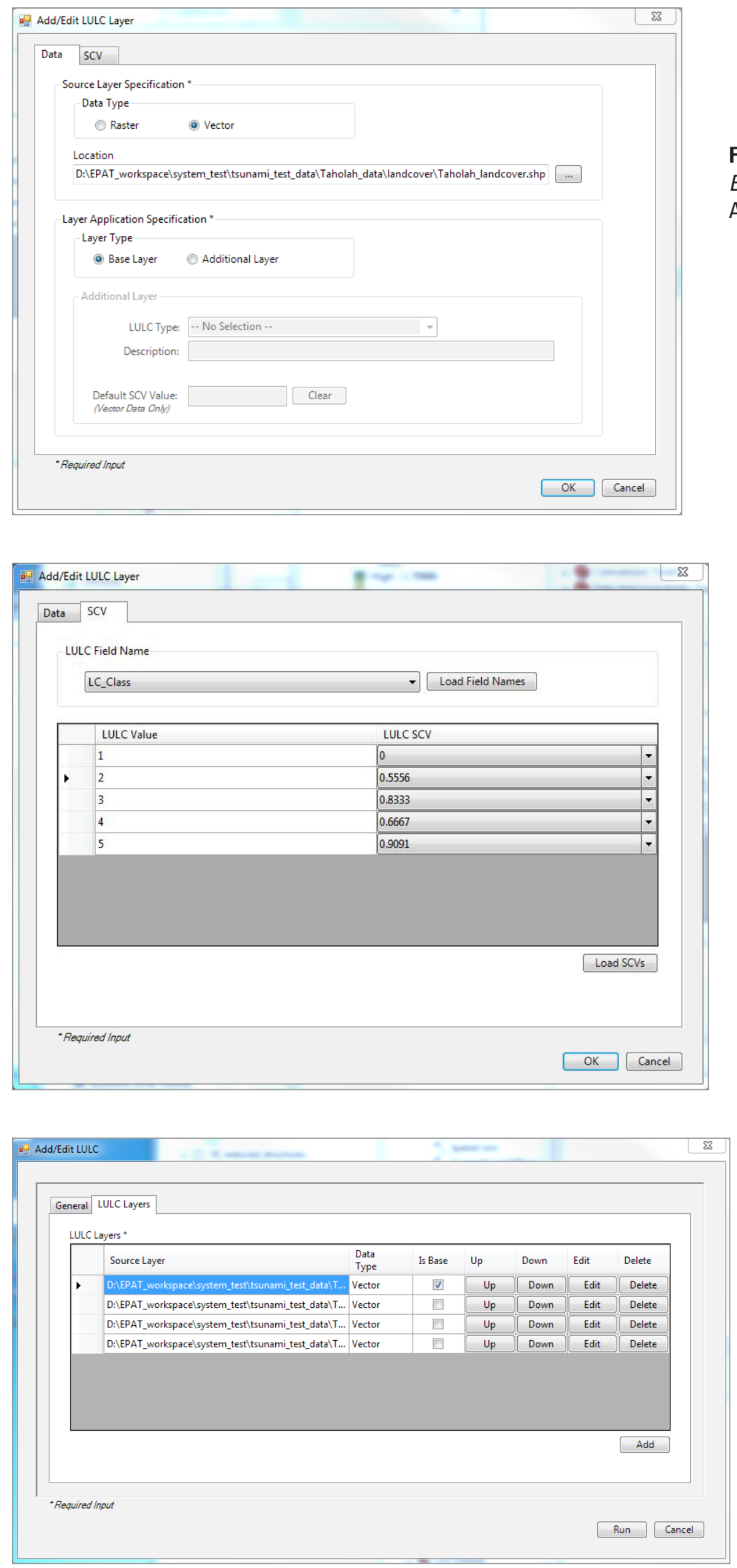

Figure 7. Screenshot of the Data tab of the Add/ Edit LULC Layer window in the Pedestrian Evacuation Analyst.

Figure 8. Screenshot of the SCVtab of the Add/Edit LULC Layer window in the Pedestrian Evacuation Analyst.

Figure 9. Screenshot of the LULC Layers tab of $A d d / E d i t ~ L U L C$ window (completed) in the Pedestrian Evacuation Analyst. 
Table 2. Typical land-cover layer ordering for the cost-inverse raster of the Pedestrian Evacuation Analyst.

\begin{tabular}{|c|c|}
\hline Order & Land-cover layer \\
\hline 1 & Base land cover \\
\hline 2 & Water \\
\hline 3 & Roads \\
\hline 4 & Buildings \\
\hline
\end{tabular}

Table 3. Default speed-conservation values (SCV) and their corresponding land-cover types (based on Soule and Goldman, 1972) in the Pedestrian Evacuation Analyst.

\begin{tabular}{|c|c|}
\hline SCV & Land-cover type \\
\hline 0 & Water, buildings, etc. \\
\hline 0.5556 & Unconsolidated beach (sand) \\
\hline 0.6667 & Heavy brush \\
\hline 0.8333 & Light brush \\
\hline 0.9091 & Developed \\
\hline 1 & Roads \\
\hline
\end{tabular}

\section{Hazard-Zone Preprocessing}

The Hazard-Zone Preprocess tool can be used to create the safe-zone polygons from an input layer of hazard zones and the study area outline. This step can be skipped if a safezone layer is already available. This tool simply erases the hazard zone from the study area to create the safe zone. It also breaks the safe zone into individual polygons to assist in the safe-zone validation step, which is a manual step described in the next section. The following steps describe hazard-zone preprocessing:

1. Click on the hazard zone preprocess (yellow triangle with !) icon (fig. 2) to open the main window, which functions in a similar manner to the main menus of the other preprocessing steps.

2. Click on the New button to open the Add/Edit Hazard Zone window on the General tab.

3. Use the AutoFill button to get a default name for the preprocessing or enter a name of choice.

4. In the Study Area field, use the drop-down list to select a preprocessed study area generated from the Preprocess DEM step.

5. Click on the Hazard/Safe Zone tab and use the ellipsis (...) button to navigate to the input (source) hazardzone vector. The name of the output (target) safezone shapefile is prefilled in the workspace folder, and this can be modified if desired. Note that this tool generates a preliminary safe-zone file, because a manual inspection or cleanup step is critically important before using this safe zone in further processing.

\section{Click on the Run button to run the tool.}

The Hazard Zone process status window is displayed signaling successful completion of the tool and reminding the user to verify the results before proceeding with the path distance calculations. The preliminary safe zone created is also loaded into the ArcMap table of contents.

\section{Safe-Zone Validation}

If a safe-zone polygon layer is derived from a set of hazard-zone polygons, the quality of the layer is dependent on the quality of the hazard-zone input file (fig. 10). For example, if the hazard boundary does not extend completely to the studyarea boundary, small safe-zone polygons will erroneously be created along the study-area edge. Additionally, if the hazard zone consists of multiple polygons that do not align exactly, the slivers between hazard polygons will also cause erroneous safe zones. Errors like these are best detected by manual inspection and hand-corrected by the user. After running the Hazard-Zone Preprocess tool, it is important to manually examine the resulting safe-zone polygons for correctness and to remove any erroneous polygons. Once this task is complete, the final quality-assured safe-zone shapefile can be used as input to the path-distance tool. It is the user's responsibility to verify the accuracy of the safe zone before it is entered into the next tool.

\section{Path-Distance Surface Creation}

The Path-Distance tool takes as input a preprocessed DEM, a least cost-inverse raster, and a validated safe zone and determines the travel distance from every cell in the study area to the nearest safe zone. It does some preprocessing of the input safe zone by checking projection, clipping to study area, and converting to a raster at the analysis cell size. When the tool has completed, the target-path distance raster is ready to be multiplied by the travel speed in the next step to determine the travel times to safety. Because the path-distance values are not meaningful until they are multiplied by the travel speed, this tool does not display the resultant raster at the end of processing. However, by keeping this computationally intensive step separate from the next step, the path-distance surface is generated once and is then available to be used with a variety of travel speeds. The following steps describe path distance surface creation:

1. Click on the Path Distance (PD) icon (fig. 2) to open the main window.

2. Click on the New button to open the Add/Edit Path Distance window on the General tab.

3. Use the AutoFill button to get a default name for the processing, or enter a name of choice. The name and location of the resultant path-distance surface is filled in automatically but can be modified by the user. 


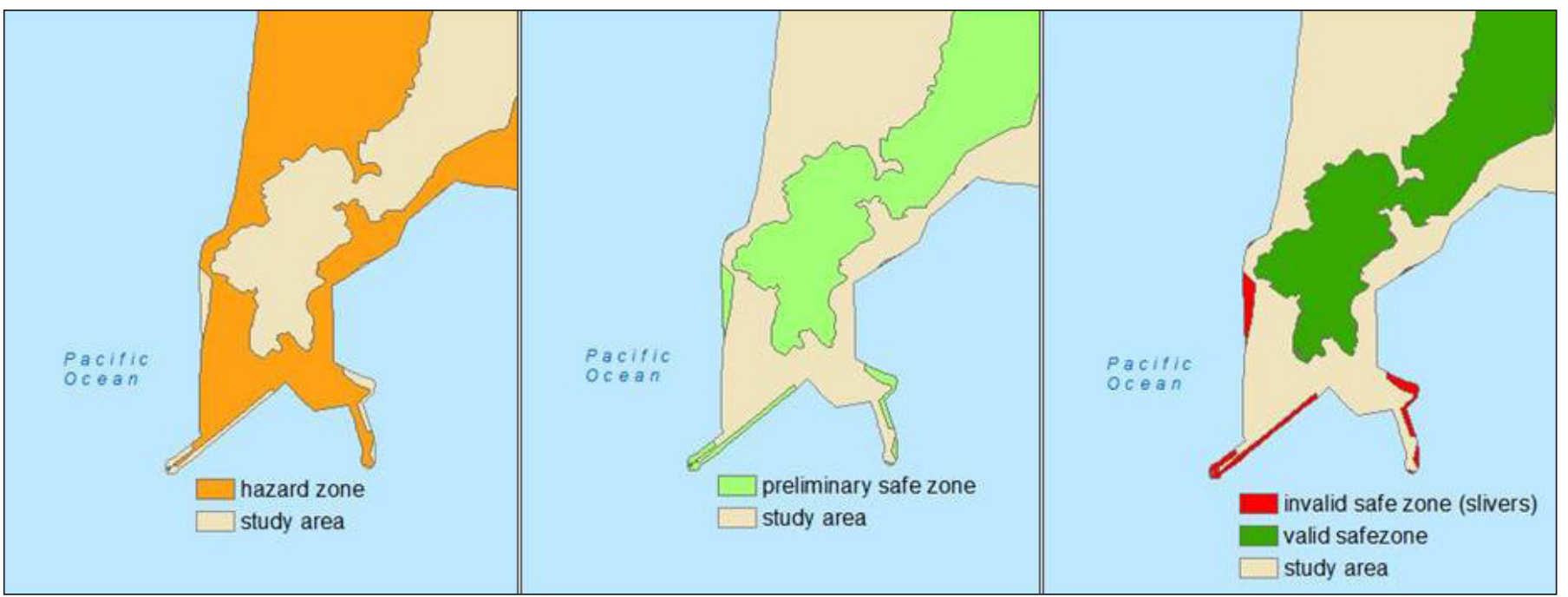

Figure 10. Screenshots of the hazard zone and study area (left), tool-derived preliminary safe zone (middle), and valid safe zone after invalid slivers are removed (right) in the Pedestrian Evacuation Analyst.

4. Click on the Preprocessed Inputs tab to enter the DEM and land-cover information.

5. Use the drop-down menus to select a preprocessed DEM layer and a preprocessed LULC layer.

6. Click on the Safe Zone tab to enter the safe zone for processing. Because this layer may have undergone some editing if created by the Hazard Zone preprocessing, there is no preprocessed safe-zone name to be referenced here. Use the ellipsis button (...) to navigate to the appropriate safe-zone file. The name of the resultant (target) safe-zone layer will be filled in automatically.

\section{Click on the Run button to start processing.}

Depending on the size of the study area and the resolution of the analysis, the path-distance processing could take some time to complete. Depending on the processor speed and amount of available memory (and ArcMap version), processing could take several minutes to nearly an hour. A progress bar will appear while the tool is running, but when processing is complete the path-distance surface will not be added to the ArcMap table of contents.

\section{Evacuation Surface Creation}

In this processing step, the target path-distance raster is multiplied by a travel speed to create the evacuation-time surface containing the travel times in minutes to safety. In a typical workflow, a single path-distance surface is created and then multiple time maps are generated at a variety of travel speeds. To functionally group the results by travel speed, a set of folders will be created within the workspace, and results folders for each processed travel speed and the tool will automatically append the speed to the names of processed surfaces during this and subsequent tool steps. An evacuation surface is created with the following steps:

1. Click on the Evacuation Surface (ES) icon (fig. 2) to open the main window and click on the New button to open the Add/Edit Evacuation Time Surface window.

2. Use the AutoFill button to get a default name for the processing.

3. Select a processed path-distance name from the Path Distance pull down menu. The name of the resultant (target) evacuation-time surface is automatically filled in. Note that the auto filled Name and the Target Evacuation Time Surface Raster do not contain any reference to travel speed because a speed value has not yet been selected.

4. Choose a travel speed from the Travel Speed Name drop-down list. A slow-walk travel assumption of 1.1 meters per second is representative of a mixed population with ranges in age and physical mobility (Wood and Schmidtlein, 2012). If one of the preprogrammed values is selected (table 4), the corresponding travel speed in meters per second is displayed in the Speed field, the travel speed is automatically appended to the processing name, and a travel-speed folder is added to the target raster file path. To enter a custom value, select "Other" in the pull down menu and type the desired value in the speed field.

5. Click the Run button to start processing and create an evacuation surface.

When processing is complete, the evacuation-surface completion window will appear and the evacuation surface will be added to the ArcMap table of contents. Before proceeding to the time-map step, the user is encouraged to 
Table 4. Travel-speed names and corresponding values in the Pedestrian Evacuation Analyst.

\begin{tabular}{ll}
\hline \multicolumn{1}{c}{ Travel-speed name } & \multicolumn{1}{c}{$\begin{array}{c}\text { Travel-speed value }{ }^{1} \text { (meters/ } \\
\text { second) }\end{array}$} \\
\hline Slow walk & 1.1 \\
Fast walk & 1.52 \\
Slow run & 1.79 \\
Fast run & 3.85 \\
Other & 0 (user enters value) \\
\hline
\end{tabular}

${ }^{1}$ Walking speeds from Federal Highway Administration (2009); running speeds from MarathonGuide.com (2011). See table 2 in Wood and Schmidtlein (2012).

examine the evacuation surface as described in the next step. The values in the evacuation surface are in minutes.

\section{Maximum Time-Value Determination}

The path-distance algorithm in ArcGIS is sensitive to abrupt changes in elevation. When analysis cell sizes are larger, such as 10 square meters or more, sudden elevation changes are smoothed out and do not affect the algorithm. However, when working with small cell sizes (for example, 1 square meter), elevation may change abruptly from one cell to the next. In this case, the path distance algorithm may compute a very large travel distance in the vicinity of the sudden elevation change. The user may wish to screen out these very high values (high time values after multiplication by the travel speed) from the final time map to concentrate on the more realistic travel times that would be faced by the population in the study area. The following description provides suggestions for manually identifying a realistic maximum travel time for the current evacuation-time surface. This maximum travel time is then entered as a parameter in the Time Map tool and all values in the time-map raster that exceed the maximum travel time are set to this maximum time.

We use ArcMap's symbology for the time-surface layer to help the user identify any unusually high travel times. In ArcMap's table of contents, right-click on the time surface raster (by default this layer is named TETS_R) and select Properties to open the Layer Properties window, then select the Symbology tab. The leftmost pane of this window has a heading of "Show:" and will most likely contain the rendering options of Unique Values, Classified, and Stretched. Click on Classified to change to the classified option and click on the Classify button on the right of the window. This will bring up the Classification window with a histogram of travel time values. Figure 11 shows the histogram for two study areas, where travel times are evenly spaced across the length of the histogram in one study area (fig. 11A) but include anomalous outliers in the other study area (fig. $11 B$ ). For the community in figure $11 B$, the user may want to set a maximum travel time such as 300 (minutes), which results in all values higher than the maximum travel time being reclassified to the maximum

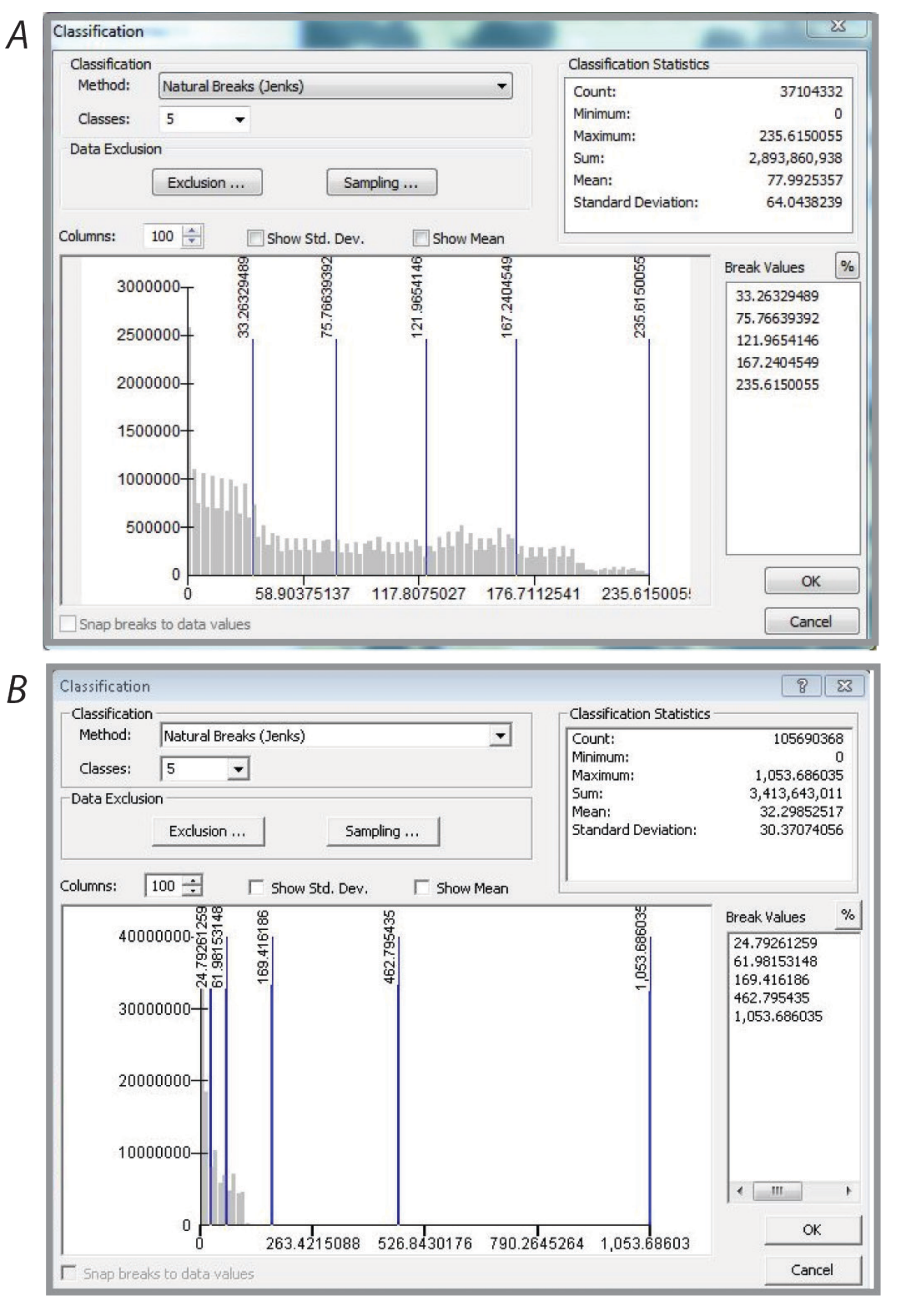

Figure 11. Screenshots of Classification windows in the Pedestrian Evacuation Analyst showing histograms for two study areas, $A$ and $B$. Note that the histogram values on the top are spaced across the length of the graph, whereas a few outliners in the histogram on the bottom cause the majority of values to be compressed on the low end of the graph.

value entered. This may aid in visualization of the final time map. Once a suitable maximum time value has been determined, it is entered by the user as a parameter for the Time Map tool. Note that this is an optional parameter in the Add/ Edit Evacuation Time Map window and can be omitted if no adjustment to the raster is necessary.

\section{Time-Map Generation}

A time map (in minutes) is generated from the evacuation-time surface by reclassifying the surface into an integer raster at 1-minute increment bands. This raster is then converted to polygons for use in the population analysis. As mentioned above, the evacuation-time surface will be reclassified to screen out values above a maximum travel time if desired. Use the following steps to create a time map: 
1. Click on the Time Map (TM) icon (fig. 2) to open the main window and click on the New button to open the Add/Edit Evacuation Time Map window.

2. Use the AutoFill button to get a default name for the processing.

3. If a maximum travel time was determined from the previous step, enter the value in the Maximum Travel Time field.

4. Choose the evacuation-time surface from the pull down list. The resultant (target) evacuation-time-map vector and raster names are automatically filled in.

5. Click on the Run button to start processing.

\section{Vertical Evacuation}

Vertical-evacuation (VE) structures, such as buildings or berms, are substitutes for naturally occurring high ground within a hazard zone. They are designed to withstand the sudden-onset hazard and provide a safe haven for nearby population evacuation. Multiple potential VE sites can be analyzed to determine the populations served by each structure, and this information can aid in the site selection decision-making process.

The Vertical Evacuation tool takes as input a shapefile of VE locations and runs a full analysis with each structure added in turn to the baseline safe zone. The VE input shapefile can be either polygons or points. If a point shapefile is entered, the user will be prompted to enter a side length for the structures, and each point will be converted to a square polygon with the specified side length. The user also has the option to enter a field name in the input shapefile that contains names or codes assigned to each structure. Because these name fields are used to identify the raster surfaces and the VE results in the graphing, the names are limited to $2-4$ characters, starting with a letter and containing only letters and numbers. If no name field is provided, a code of VE1, VE2, and so on is assigned to each structure.

For each VE structure, the polygon is merged with the baseline safe zone and then the path distance, evacuation-time surface, and time-map processing are executed for the study area. The resulting surfaces for each VE location are stored in their own folders in the results area. The following steps outline VE processing:

1. Click on the Vertical Evacuation (VE) icon (fig. 2) to open the main window and click on the New button to open the Add/Edit Vertical Evacuation window.

2. Use the AutoFill button to get a default name and select an evacuation-time map from the dropdown list. This time map becomes the baseline map for the VE time maps.
3. Click on the Vertical Evacuation Vector tab (fig. 12) to enter the VE shapefile.

4. Use the ellipsis (...) button to navigate to the shapefile containing VE points or polygons.

5. Once the File Path field is complete, the Identification Field contains a drop-down list of attribute table text fields in the shapefile. Only text fields are valid source fields for this name in the attribute table. If one of the fields is a unique identifier for each structure, select the field here. (Name/code must start with a letter, contain only letters or numbers, and have a length of 2-4 characters.) Otherwise, leave this field blank and the structures will be named in the order they appear in the attribute table.

6. The Target Vertical Evacuation Layer Directory field has been prefilled with the folder location where the VE surfaces will be stored.

7. Click on the Run button to start processing.

If the input shapefile is a point file, the Point Features Detected window will appear to verify that the points are to be buffered and converted to polygons. Click Yes and the Convert Points to Polygons window appears with a target vector file name preselected. In the Edge Distance box, enter the side length to be used to create the square polygons centered on the input points. Click $O K$. The polygons will be created and the VE processing will begin automatically.

Processing multiple VE structures will take some time to complete, depending on the number of structures and the size of the study area. To estimate the time required for processing VE structures, observe the time required for path-distance, evacuation-time-surface, and time-map processing of the baseline time map, and multiply this by the number of VE structures. A progress bar will be displayed during processing. Figure 13 shows an example of the baseline time map for Taholah, Washington, along with the time maps generated with three VE structures in a tsunami-hazard zone.

This tool uses one VE structure at a time to merge with the safe zone and generate a new travel-time map. To generate a time map for multiple VE structures at the same time (such as VE 1 and VE 2 for Taholah), use the Safe-Zone Merge (SZM) tool described next to select multiple VE structures to merge. The new safe zone can then be used as the basis for new processing.

\section{Safe-Zone Merge}

The optional Safe-Zone Merge tool merges the original safe zone with a VE structure polygon using ArcGIS's Union tool. This tool is not a required step in the processing workflow and can be skipped for most scenarios. It may be used to create a new, permanent safe zone with one VE structure in place or with multiple VE structures in place to examine 
their interaction. With this new safe zone, the user can create a new scenario for additional processing. The following steps describe the merging of safe zones:

1. Click on the Safe-Zone Merge (SZM) icon (fig. 2) to open the main window and the New button to open the Add/Edit Merge Safe Zone window on the General tab.

2. Use the AutoFill button to have a name preselected in the Name field and click on the ellipsis (...) button to navigate to the safe zone vector. A name and location for the resultant merged safe zone shapefile is automatically filled in.
3. Click on the Vertical Evacuation tab to choose the VE structures for merging. During Vertical Evacuation processing, each VE structure is copied to a separate shapefile and stored with the result surface. By selecting the VE processing name from the drop-down list, the names and file paths of each VE structure are filled into the Vertical Evacuation Layers table.

4. Click in the checkbox in the Include column to select the structure(s) to merge. All checked structures will be merged into the same new safe-zone file along with the original safe-zone polygons.

5. Click on the Run button to start processing.
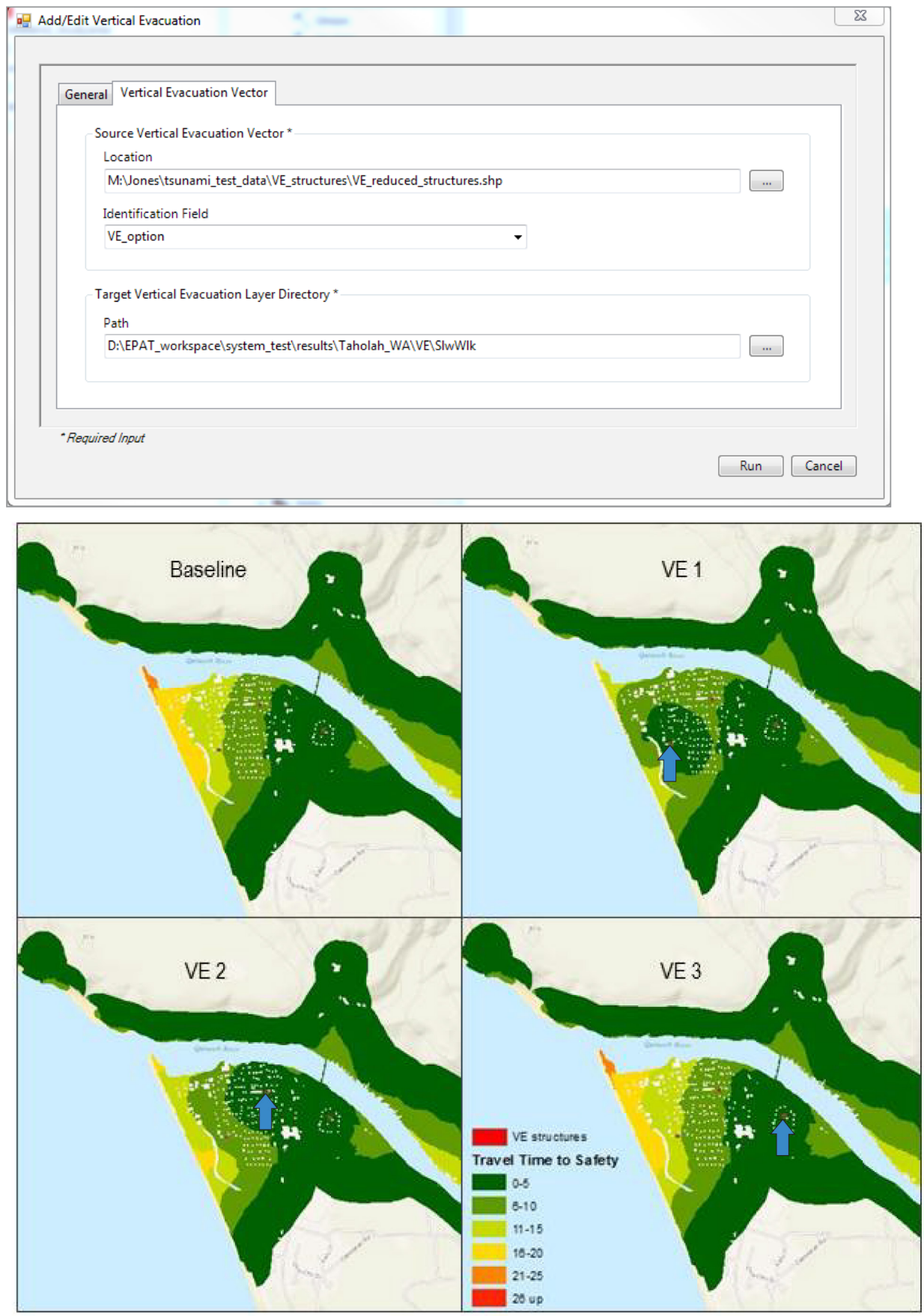

Figure 12. Screenshot of Vertical Evacuation tab of the Add/Edit Vertical Evacuation window in the Pedestrian Evacuation Analyst.
Figure 13. Screenshot from the Pedestrian Evacuation Analyst comparing time maps for baseline processing and vertical evacuation (VE) structures for Taholah, Washington. VE structures are indicated by the blue arrows. 
When the shapefiles have been merged, the file is added to ArcMap's table of contents and the completion window is displayed. A good workflow might be to create a new portfolio for processing of a new baseline time map with this merged safe zone.

\section{Population}

The Population tool uses input population data and the processed time map to determine the numbers and types of populations at various travel times to safety. The tool is set to accept five different population types - residents, employees, public venues, community services, and dependent-care facilities. It also has an optional "other" population category that can be defined by the user; for example, the user may want to include occasional tourist populations, such as tourists related to a cruise ship or tourists in town for an annual festival or other special event. Population shapefiles can be entered in either point or polygon form for evaluation against the time map (population polygons will be converted to points inside the input polygons.) An optional polygon shapefile containing jurisdictional information, such as city and county boundaries, can also be added. The tool uses this layer, applied across the study area for all population files, to break each time step down by geographic entity and report population by time and location. Preprocessing of the input population shapefiles occurs in this step as well, with each shapefile checked for correct projection and clipped to the study area. On tool completion, the processed population files are added to the ArcMap table of contents, and the text files (in .csv, or comma-separated values, format) of times and counts for each population type are written to the results folder.

The Pedestrian Evacuation Analyst is designed to count population only within the hazard zone. The minimum expected travel time to safety is 1 minute (fractional travel times are rounded up to the next whole number value). Travel times of zero may occur for population points very near to the safe zone. The Population tool checks for values of zero in the processed population file and converts these to one (1) as the population values are tallied in the tables.

The following describes the population processing steps:

1. Click on the population (two head-and-shoulder images) icon (fig. 2) to open the main window, and click on the New button to open the Add/Edit Population window on the General tab.

2. Use the AutoFill button to get a default name for the processing.

3. Select a preprocessed study area from the drop-down list in the Study Area field. The study area is used for population-file preprocessing to check projection and clip input files to the study area.

4. In the Baseline Evacuation Time Map area, select a processed time map from the drop-down list.
5. Click on the Population Preprocessing tab to add the population-data layers (fig. 14).

6. To add a jurisdictional boundary layer for counting population by location, use the ellipsis (...) button in the Community Boundaries Vector area to navigate to the file. The Community Boundaries Field will populate with all the text fields in the jurisdictional layer; choose the text field that contains the boundary names. This jurisdictional layer is optional, and population data can be processed without jurisdictional categories.

7. To add a population layer, click on the $A d d$ button in the Source Population Layers area, and the Add/Edit Population Layer window will appear (fig. 15). This window is used to enter information about each individual population type. Use the ellipsis (...) button to navigate to the input file.

8. In the Population Type area, use the drop-down list to select a population category or choose "Other" to enter a new category. The main population types are residents, employees, dependent-care facility, community support facility, and public venue. To enter a new category such as schools, select Other and type in "schools" in the Name field.

9. Click on the Population Layer Fields tab of the Add/ Edit Population Layer window (fig. 16) to open the table containing a list of numeric fields from the input file.

10. Click in the checkbox in the Include column next to the field(s) that contains population-count information. Because field names are often not very descriptive, the Field Alias column is available for the user to enter a more descriptive column name. This alias name will appear in the population count tables.

\section{Click $O K$.}

12. Once all the population layers are entered, click on the Population Table Generation tab to enter the final information for processing (fig. 17).

13. In the Target Population Tables Directory field, a default location for the population tables is automatically filled in.

14. If no vertical-evacuation structures have been processed for population analysis, leave the Vertical Evacuation section blank. If vertical-evacuation time maps are to be evaluated along with the baseline time map, use the Name field drop-down list in the Vertical Evacuation area to select the baseline time map, and the VE surfaces for this baseline will be loaded into the layers table. Check the Include box for the VE time maps to use in population processing. 
15. Click on the Run button to start processing.

16. To facilitate the quick generation of population tables for different travel speeds within the same study area, the population-processing step contains a shortcut for data entry. After one set of population tables has been generated using the New button from the Process Population main window, additional travel-speed population processing can be expedited by clicking the Edit button on an existing population name and selecting a different time map from the Baseline Evacuation Time Map drop-down menu (this feature is not available in all processing steps). This will update the Name field on the General tab and the travel speed folder names on the other two tabs. The
Source Population Layers and Source Community Boundaries Layer will already be filled in, as well as any selected VE structures. The VE structures can be removed if desired by setting the Name field in the Vertical Evacuation area to "No Selection."

17. If an existing population name is being edited and possibly modified as described in the last paragraph, a window will appear asking if a new population name is being added or if a current name is simply being updated. Click the $A d d$ button if a new population name with a different travel speed has been created by editing an existing name. The Update button simply proceeds with processing under the same population name.
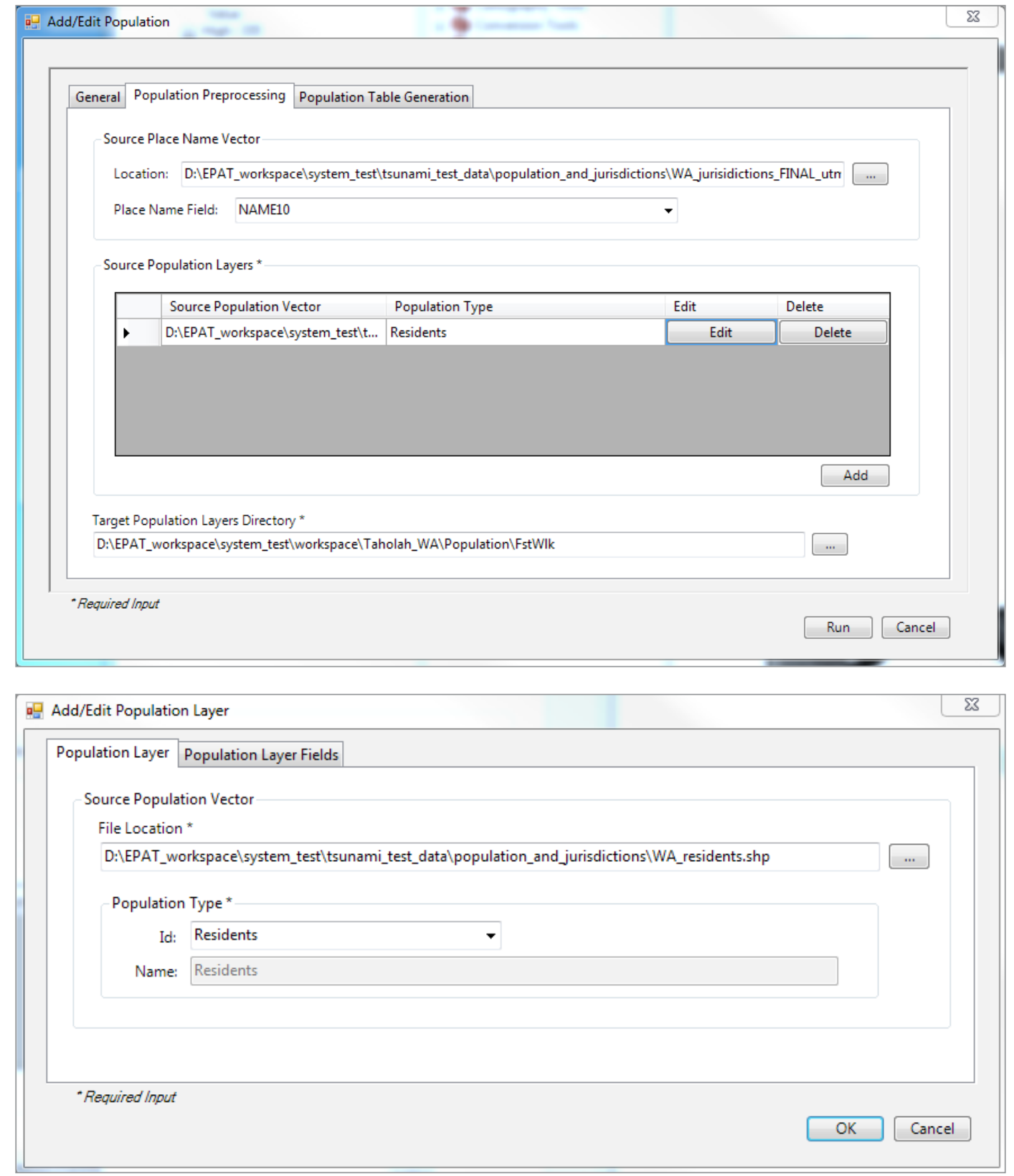

Figure 14. Screenshot of the Population Preprocessing tab of Add/Edit Population window in the Pedestrian Evacuation Analyst.
Figure 15. Screenshot of the Population Layer tab of Add/Edit Population Layer window in the Pedestrian Evacuation Analyst. 


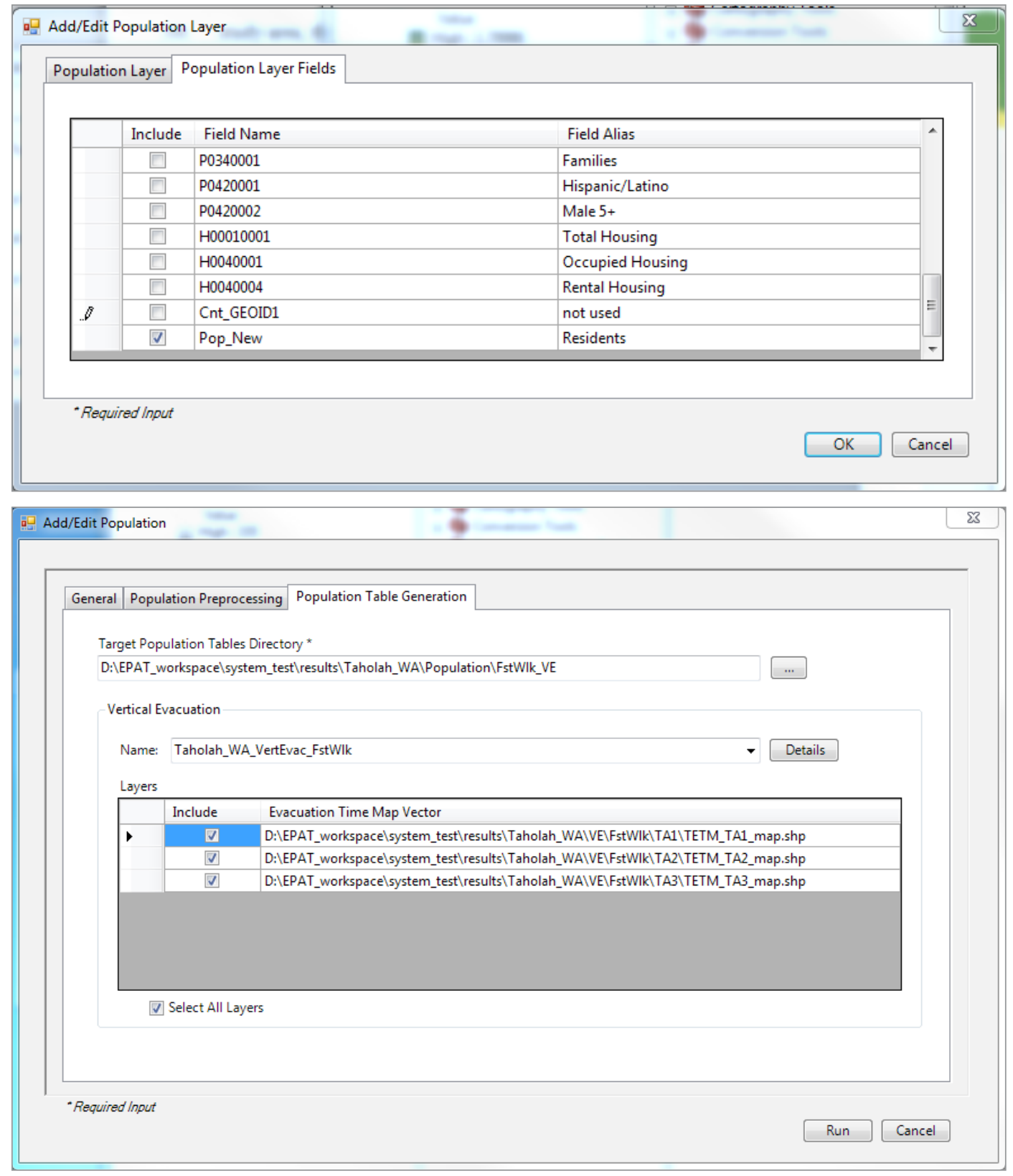

Figure 16. Screenshot of the Population Layer

Fields tab of Add/ Edit Population Layer window in the Pedestrian Evacuation Analyst.

Figure 17. Screenshot of the Population Table Generation tab of Add/ Edit Population window (vertical-evacuation options selected) in the Pedestrian Evacuation Analyst.

A processing window will appear indicating the processing progress. On completion, the processed population files will be added to the ArcMap table of contents. These processed shapefiles are named by the population type with the word "identity" appended. A shapefile will be created for each input population type that has points within the study area.

The population tables are created in the designated folder and are named after the population type entered for each file. Two comma-separated values (.csv) files are created for each population type, a sparse table containing only the times with nonzero population counts, and an expanded table with a row for every time step and zeroes filled in when no population was counted at a time step. The expanded table is provided for ease of graphing and charting in an application such as Microsoft Excel to allow different population types to be combined in a single graph. Also, when a jurisdictional layer is included, the expanded table contains not only every time step but also every unique place within each time step to ease graphing of the data. The first column, labeled "Time to safety," contains the travel-time step. If a community boundaries layer was entered, the next column is labeled "Location" and contains the location name for the given count. The remaining fields are the field aliases and counts for all fields selected from the Population Layer Fields list for this population type. These .csv files are used by the Charts and Graphs tool and are also available directly for the user if custom graphs are desired.

The tool creates a variety of folders to help organize the processing results. The .csv files for the baseline time map will be under the Population folder, whereas the .csv files for each VE structure will be in the VE structure's folder within the VE upper-level folder. See appendixes B and C for information on the tool's folder system and naming conventions. 


\section{Charts and Graphs}

The Charts and Graphs (bar-graph image) tool (fig. 2) is designed to help visualize the population data in relation to travel times. The population data is stored in its own database, and when the tool is run for the first time a window will appear asking to create the Pedestrian Evacuation Analyst Charts database. Click Yes, and the database is created in the same folder as the primary Pedestrian Evacuation Analyst database.

When the database is created, the main Charts window (fig. 18) will appear and the Population list box will contain all the processed population names for the current portfolio. The Chart Type drop-down list contains the names of five different charts:

- Population Count vs Time To Safety, which is best when looking at a single population type across multiple jurisdictions in a study area;

- Population Count by Type vs Time To Safety, which is best when looking at multiple population types within a single jurisdiction or study area;

- Population Count As a Function of Travel Speed, which is useful for comparing population-exposure estimates based on multiple travel-speed assumptions;

- Population Percentage to Reach Safety, which is useful for examining changes in the percentage of a population type that can reach safety as a result of vertical-evacuation strategies; and

- Vertical Evacuation Strategy Comparison, which is useful for comparing multiple vertical-evacuation strategies with a relative comparative metric.

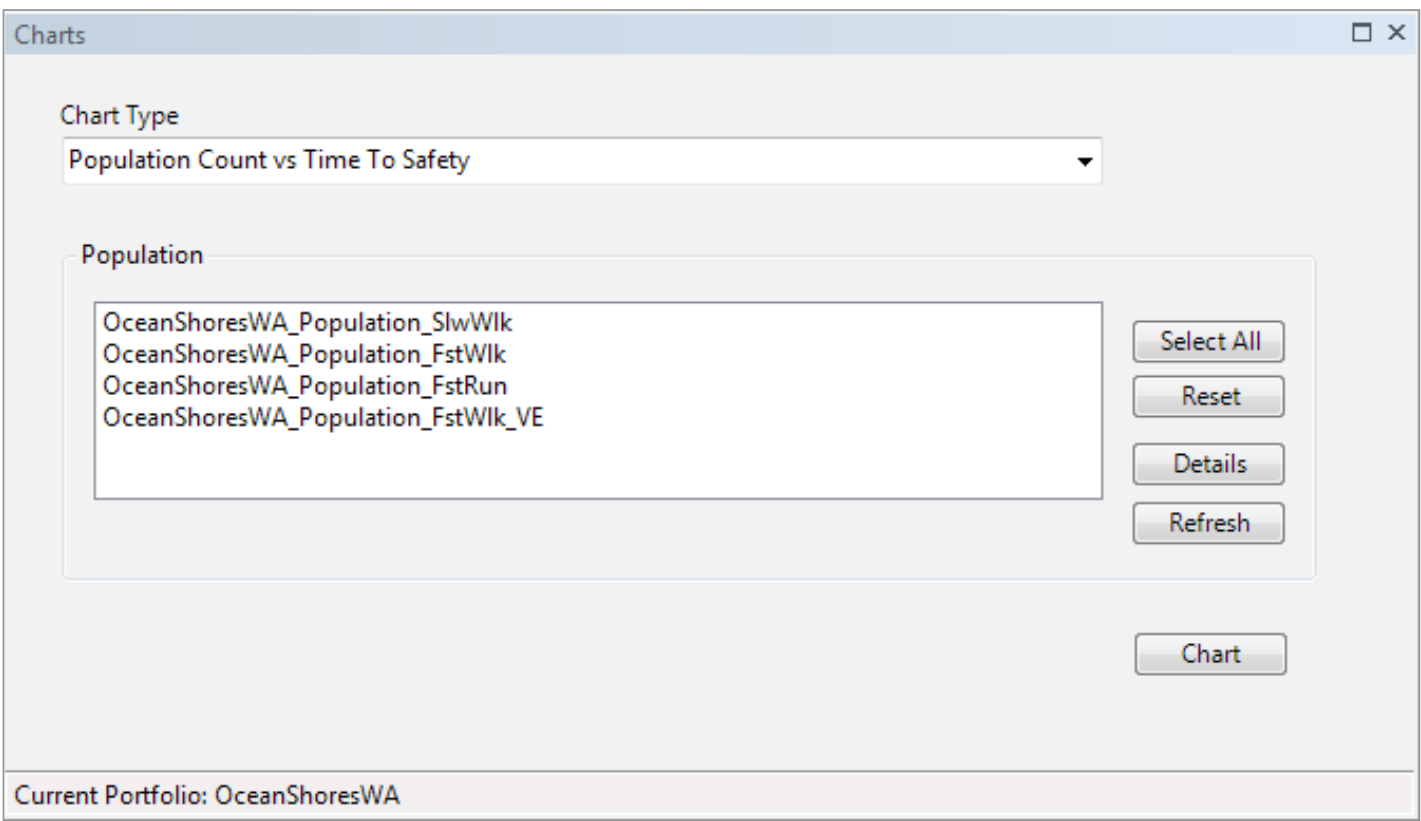

To run a chart, select a chart type from the Chart Type drop-down list and select one or more population names from the Population names list box, then click the Chart button to launch the corresponding chart form. Chart output generated from a chart form is displayed in a separate window called the Chart Viewer (fig. 19). For all chart types, the Chart Viewer lists the available output in the table of contents area and the actual chart images in the view area. The viewer can be set to display charts in one of three different view modes - single, double, or quad, allowing for as many as four charts to be viewed simultaneously. The view mode is set to single by default when the viewer is launched. Use the Single, Double, and Quad chart view buttons in the toolbar menu to switch between modes. When the view mode is set to single, a chart can be viewed simply by clicking it in the table of contents. To view more than one chart at a time, select either the double- or quad-view mode. Next, click and drag a chart from the table of contents to any pane showing in the chart view area. Repeat this step to add other charts to the viewing area.

To enlarge the chart viewing area, click and drag the split-panel divider to the left or simply use the Collapse Charts and Expand Charts buttons in the toolbar menu to collapse or expand the table of contents area, respectively. To save or print a chart, select a chart in the table of contents, then click either the Save or Print button and perform the action in the corresponding dialog window that appears.

\section{Population Count Versus Time to Safety Chart}

The Population Count vs. Time To Safety chart plots population count in relation to time to safety for a given population type. The chart provides a visual perspective of how population counts are distributed across time, identifying at a glance populations that may be unable to reach safety before the arrival time of a tsunami wave. With its chart form opened (fig. 20), select a population type from the corresponding
Figure 18. Screenshot of the main Charts window in the Pedestrian Evacuation Analyst. 

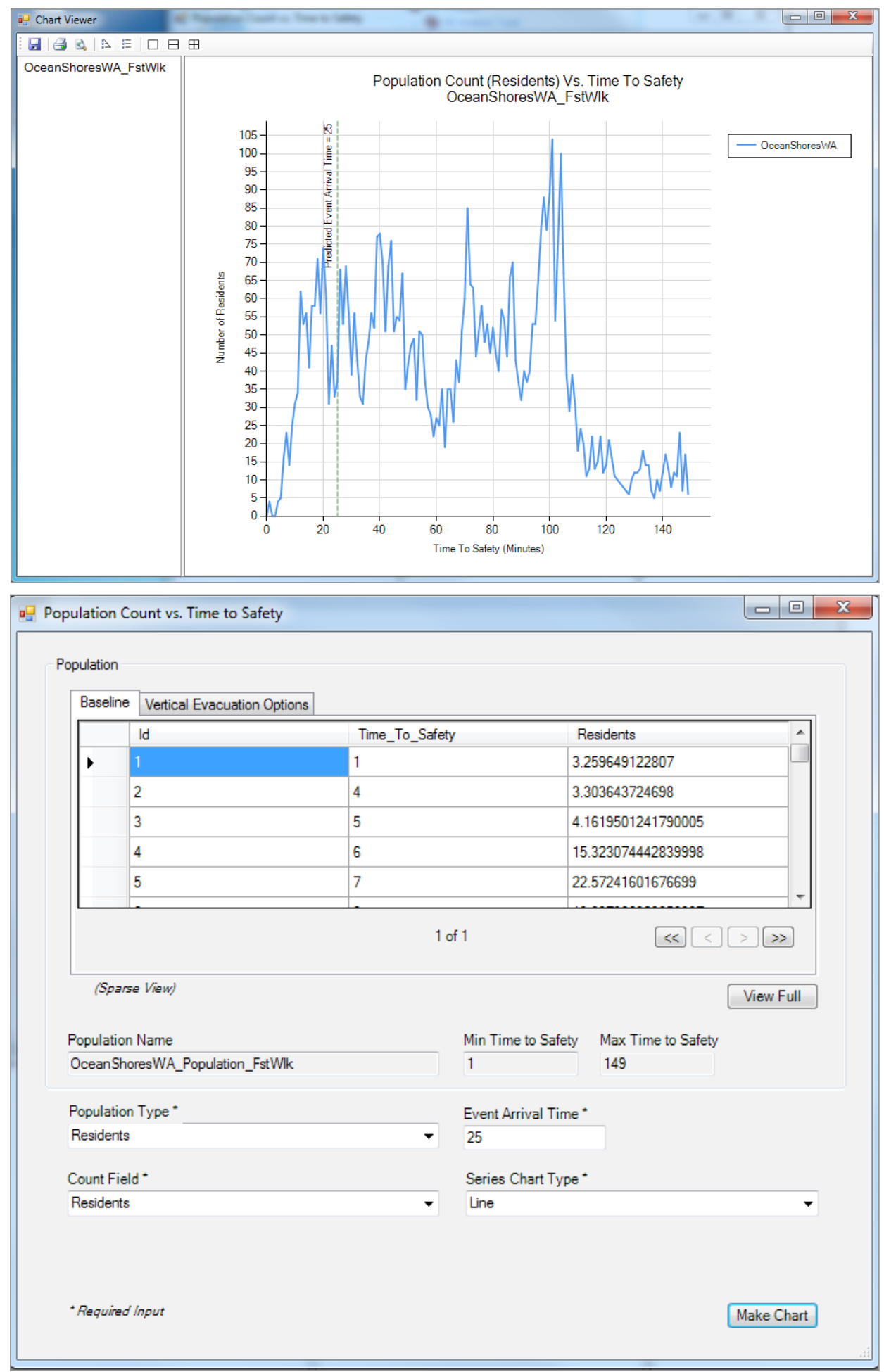

Figure 19. Screenshot of the Chart Viewer window in the Pedestrian Evacuation Analyst.
Figure 20. Screenshot of the Population Count vs. Time to Safety window in the Pedestrian Evacuation Analyst. drop-down list. Population baseline and vertical evacuation data for the selected population type will appear in a grid table located inside the Population view area.

Navigation controls located just below the grid table can be used to navigate through the baseline or the VE record set, depending on which set is currently in view. Another button located below the navigation controls allows the content view to be toggled among three choices - sparse, full, and totals. Sparse view displays records of each combination of time to safety and location for each reported time to safety value. Full view displays records of each time to safety and location combination for each time to safety value within the reported time to safety minimum and maximum value range. Totals view displays the count field totals by time to safety.

Next, specify the Event Arrival Time (expressed in minutes), then set the Count field and Series Chart Type options from their corresponding drop-down lists. When ready, click the Make Chart button to begin charting. 
When finished, the chart output will be displayed in the Chart Viewer (fig. 19), described in the general Charts and Graphs section above. A chart is generated for each selected population name and its corresponding VE options, if any. If the population data includes jurisdictional names, then the data is subdivided and graphed by jurisdictional name, and these names appear in the graph legend. This chart provides a visual perspective of the population distribution for the selected population type in relation to travel time to safety. For example, figure 19 shows how there are approximately 78 people at locations that would require 40 minutes to reach high ground at a fast walk.

\section{Population Count by Type Versus Time to Safety Chart}

The Population Count By Type vs Time To Safety chart plots the aggregated population count for the included population types in relation to time to safety. Because this chart is plotting multiple population types (such as residents, employees, and tourists), if more than one population name is selected for comparison, then only the population types that
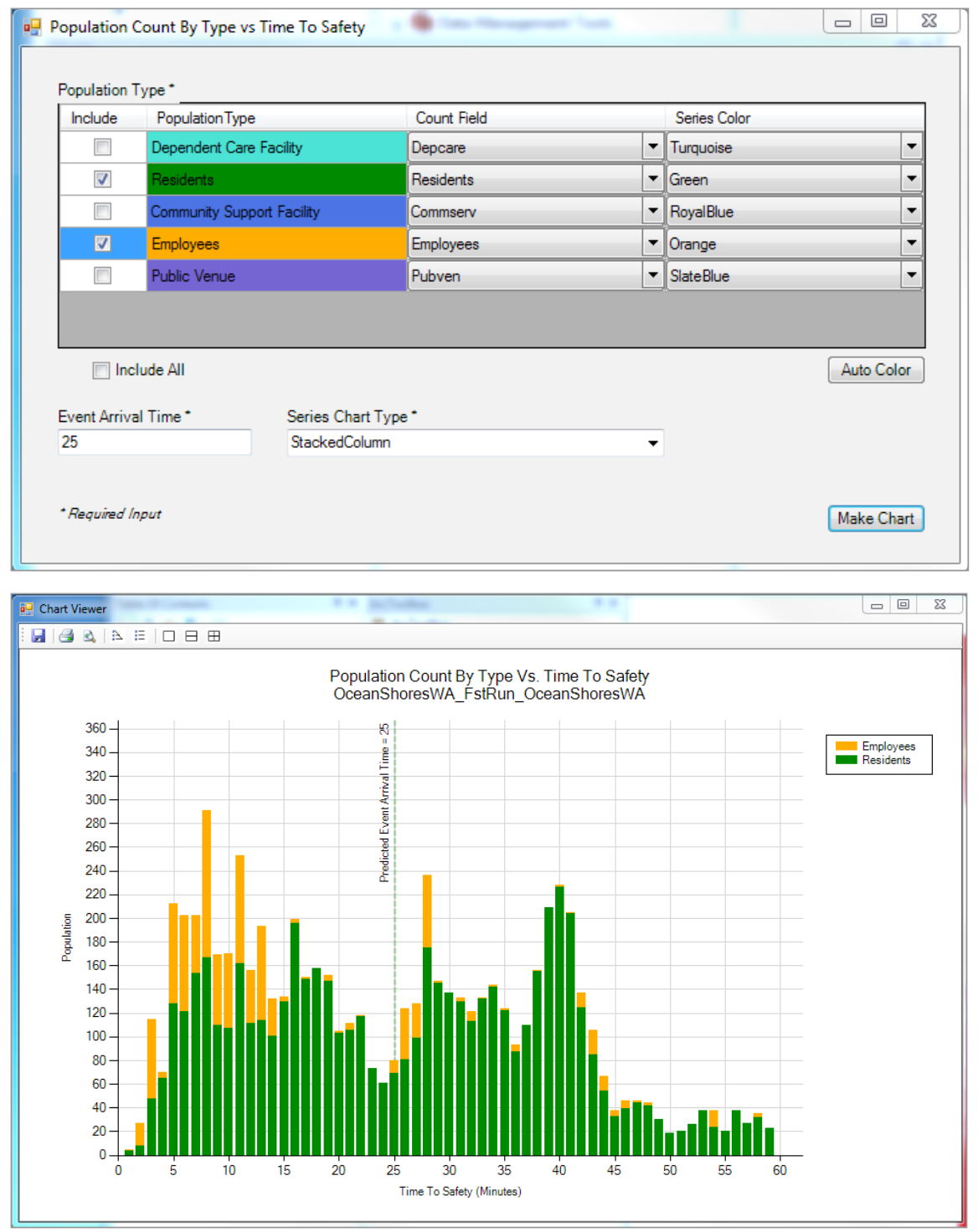

are common to each selected population name are included for charting. The chart window (fig. 21) contains a Population Type table showing the common population types along with their respective count fields. For each population type, set the Count Field and Series Color values from their respective drop-down lists. Next, specify the Event Arrival Time and select a series chart type option from the Series Chart Type drop-down list.

Click the Make Chart button to begin charting. When finished, the chart output will appear in the Chart Viewer (fig. 22). A chart is created for each selected population name and any corresponding VE options. The chart legend identifies the population types by selected color. This chart shows all population types on the same graph for comparison. When the Line series chart type is selected, each individual graph is overlaid on the same chart. When the StackedColumn chart type is selected, the cumulative totals at each travel time are displayed. This chart provides both a view of the different population type distributions in relation to each other, as well as an overall population distribution (fig. 22).
Figure 21. Screenshot of the Population Count By Type vs Time To Safety window in the Pedestrian Evacuation Analyst.
Figure 22. Screenshot of a Population Count By Type Vs. Time to Safety chart in the Pedestrian Evacuation Analyst. This example chart for Ocean Shores, Washington, shows a combined population of residents and employees at each time step with, for example, approximately 240 residents and employees who would be 28 minutes from high ground at a fast run (approximately a 7-minute mile). 


\section{Population Count as a Function of Travel Speed Chart}

The Population Count As a Function of Travel Speed chart shows a comparison of a population group's ability to reach safety at different travel speeds before the arrival of a tsunami event. On the Data tab of the chart window (fig. 23), the population names are assigned into groups for graphing. The groupings are available for displaying population results by travel speed. As many as five groups can be defined. To place a population name into a specific Travel Speed group, select a Travel Speed tab and then select a population name from the Population list and click the ">" button to move the population name over to the Travel Speed group. Repeat this step to move the other population names over into their respective groups. To remove a population name from a particular Travel Speed group, select the group, then select the population name from that group and click the " $<$ " button to move the population name back into the Population list.

With the population groupings defined, select a population type and a count field from their corresponding dropdown lists. Next, specify the event arrival time (expressed in minutes). Optionally, select the Chart Vertical Evacuation Options checkbox to create charts for VE option data in addition to the baseline data. This chart requires that all processed population names included for charting contain the same jurisdictional names (if used) and contain the same set and number of VE options (if used).

The Chart Settings tab allows for customizing various chart options. The chart name for the baseline chart can be set using the Baseline Chart Name field. Similarly, a prefix string can be appended to the names of the VE option charts using the Vertical Evacuation Option Chart Name prefix field. Additionally, the Chart Title, Chart Subtitle, and Legend Title fields can be used to specify a chart title, subtitle, and legend title, respectively. Finally, the text fields in the Group Labels section can be used to assign meaningful names to the corresponding groups.

Click the Make Chart button when ready to begin charting. When finished, the chart output will be displayed in the Chart Viewer (fig. 24). A chart is generated for the baseline population data and optionally for the VE data, if the Chart Vertical Evacuation Options check box was selected. If population data included jurisdictional names, then a data series is created and plotted for each place name. Otherwise a data series is created for the designated study area of the corresponding population.

\section{Percentage of Population to Reach Safety Chart}

The Population Percentage To Reach Safety chart compares baseline and vertical evacuation option results in terms of their percentages of population reaching safety before the arrival of a tsunami event for a specified population type. In the chart window, select a population type from the Population Type drop-down list. Population baseline and VE data for the selected population type will appear in the Population view area. Controls for navigating the baseline and VE option record sets and for toggling through content view choices are provided below the grid. Next, set the values for the count field, event arrival time, and series chart type in their corresponding fields. To specify a custom color for the data, select an option from the corresponding Series Color drop-down list.

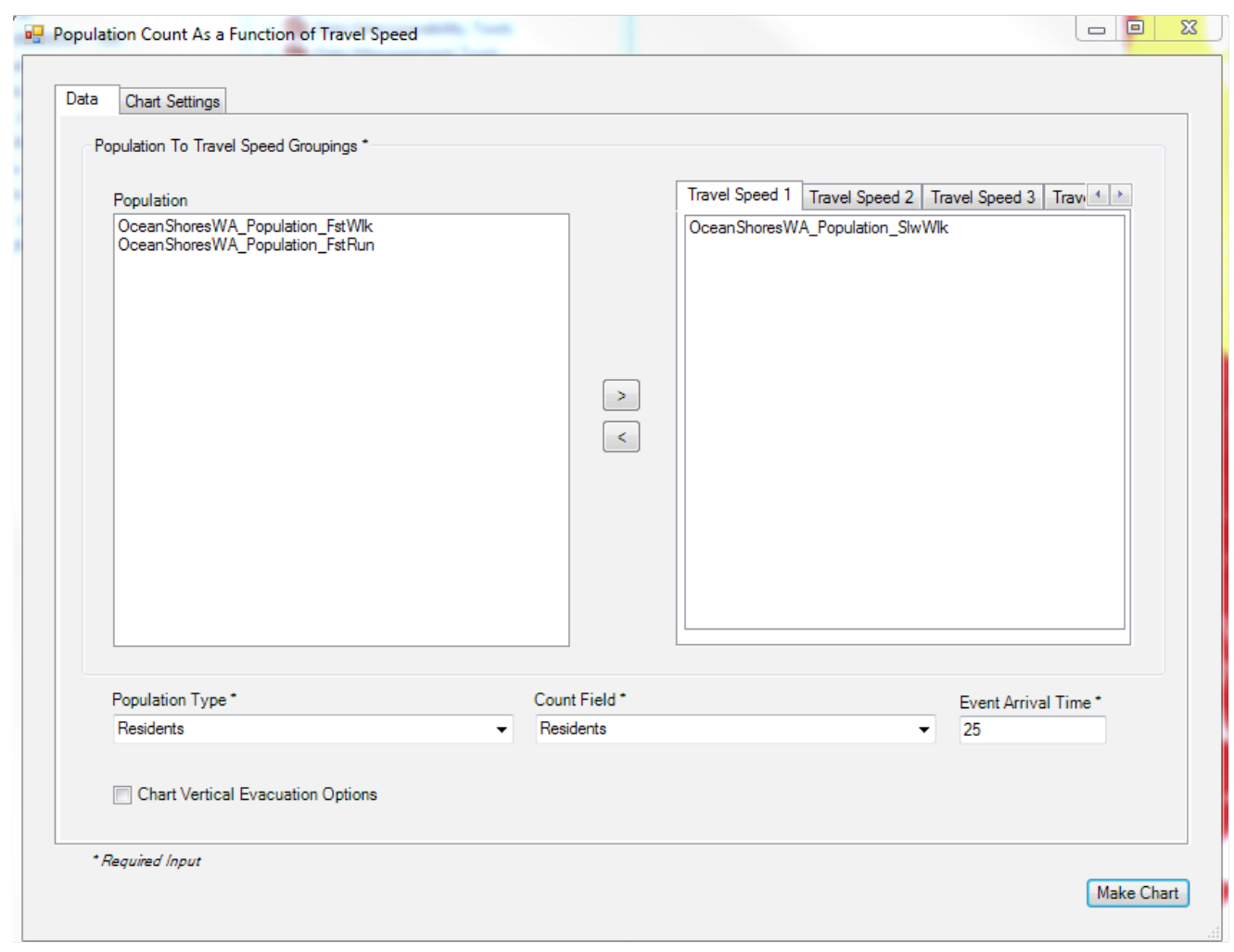

Figure 23. Screenshot of the Data tab in Population Count As a Function of Travel Speed window in the Pedestrian Evacuation Analyst. 


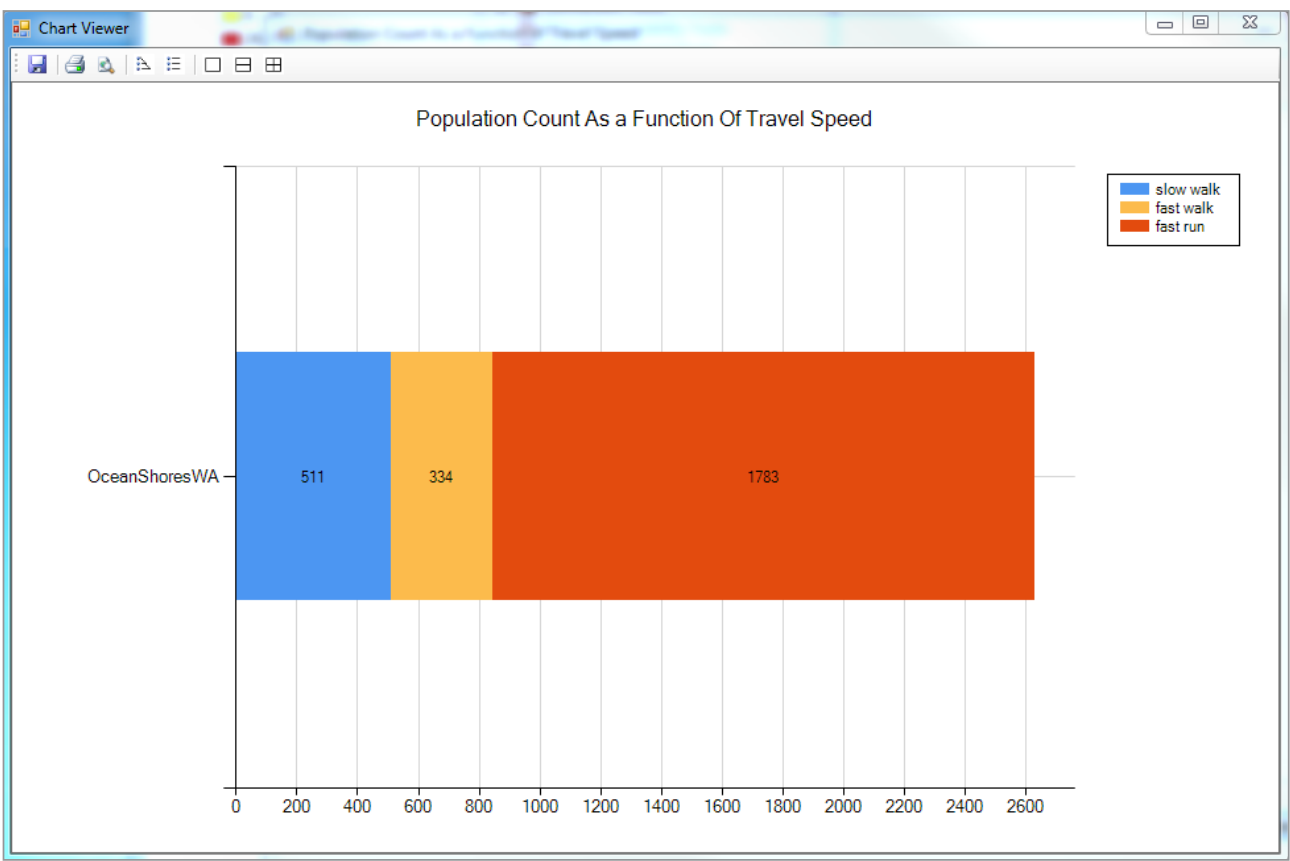

Figure 24. Screenshot of a Population Count As a Function Of Travel Speed chart results in the Pedestrian Evacuation Analyst. This example chart for Ocean Shores, Washington, shows the group values for slow-walk, fast-walk, and fastrun travel speeds assigned and plotted for a given community. It shows 511 residents able to reach high ground moving at a slow walk, whereas an additional 334 could reach high ground if the group moved at a fast walk, and an additional 1,783 could reach high ground before the event arrival if the group moved at a fast run.

Click the Make Chart button to begin charting. When finished, chart output will appear in the Chart Viewer (fig. 25). Charts are created for each selected population name. If a population name included jurisdictional information, a chart is created for each location. In each chart, a data series for the baseline percent is represented by a line that spans the chart area. Similarly, a data series for each VE-option percent is plotted using the specified series chart-type option to provide a relative comparison to the baseline percent. For example, in figure 25, approximately 15 percent of the at-risk population in the community currently may be able to reach high ground at a fast walk. This percentage increases to 37 percent for the first VE option (OS6), 30 percent for the second VE option (OS8), and so on.

\section{Vertical Evacuation Strategy Comparison Chart}

The Vertical Evacuation Strategy Comparison chart compares the VE options for a given population name in terms of a comparative normalized index. Normalized percentages are derived by first calculating the maximum population count among the VE options for a given population type. The percentage of a jurisdiction's population that could be served by a specific VE strategy is then divided by the maximum percentage. This transforms each VE percentage to a ratio relative to the maximum count found in the study area. This transformation is done for each population type to be compared. The normalized population-type percentages are then plotted against a scale that ranges from zero to the total number

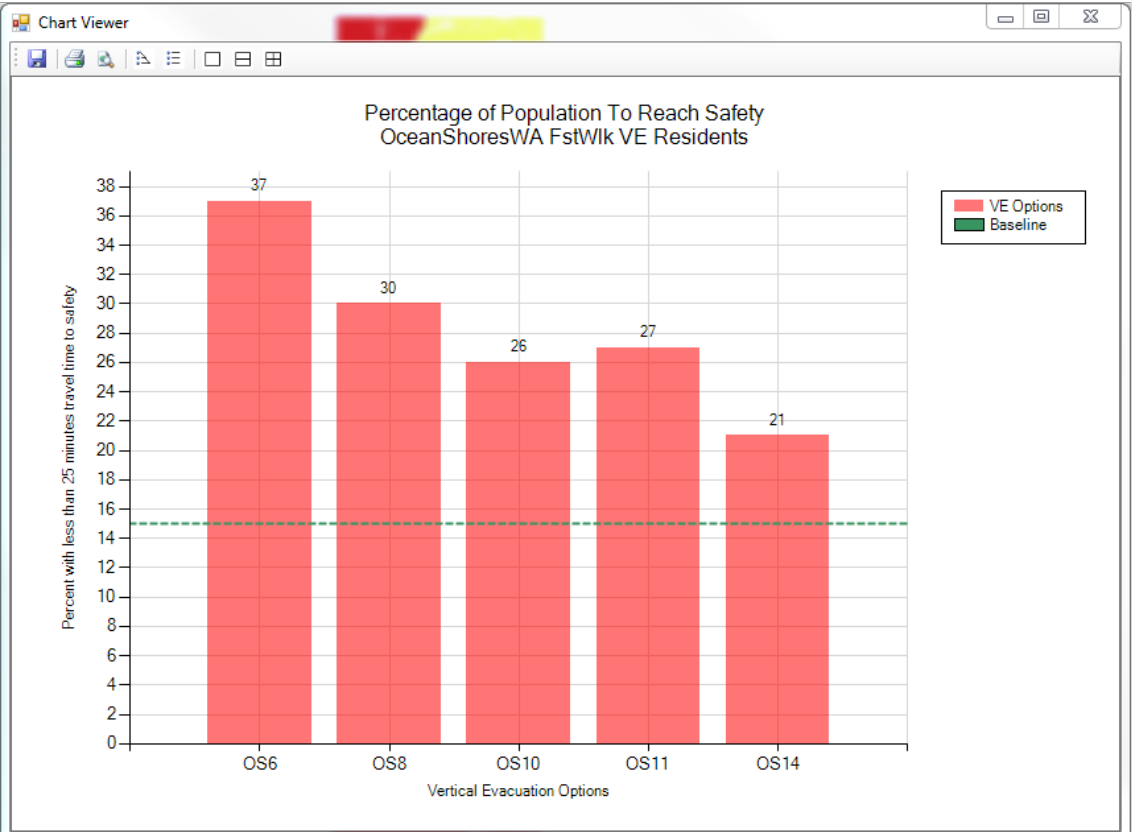

Figure 25. Screenshot of Population Percentage To Reach Safety chart results for Ocean Shores, Washington, in the Pedestrian Evacuation Analyst. 
of population types. For example, if three population types (residents, employees, and public venues) are included, then the final comparative metric would have a range from zero to three. This transformation is needed so that population types with different ranges can be compared; for example, resident counts are total number of individuals, whereas public venues are the total number of identified locations.

The chart form contains a grid table displaying the population types common to all the selected population names. For each population type, the count field and series color must be set to an option from their corresponding drop-down lists. Next, set the event arrival time and the series chart-type option.

Click the Make Chart button when ready to begin charting. When finished, chart output will be displayed in the Chart Viewer (fig. 26). A chart is created for each of the selected population names. In each chart, a data series is created for each VE option representing the normalized population type percentages. These points are plotted against a scale ranging from zero to the total number of population types processed. Each chart provides a perspective of how well a VE option performed as a whole relative to others in terms of maximizing population safety. For example, figure 26 demonstrates how vertical-evacuation option OS6 serves the greatest number of residents, employees, community services, and dependent-care facilities, expressed by it having a value of 1 for those categories. Only site OS10 serves more public venues (with a score of 1) but with far fewer residents and dependent-care facilities.

\section{Summary}

The Pedestrian Evacuation Analyst is designed to provide (1) evacuation potential maps of travel-time estimates and (2) tables of at-risk populations with travel times to safety. The software can also aid in decision support in the evaluation of vertical-evacuation options. It has potential uses for mapping sudden-onset hazards such as near-field tsunamis, volcanic lahars, and flash floods. Potentially, any application seeking to model pedestrian travel times across a landscape from a hazard zone to a safe zone may find this software useful.

The software provides features such as automated preprocessing of input data and logically grouped file management (portfolios) to keep track of files associated with different research scenarios. The software also provides the ability to (1) generate time maps at different travel speeds, (2) generate time maps for potential vertical-evacuation options and compare results against a base map, (3) map multiple populations of residents, employees, public venues, community services, and dependent-care facilities, and (4) determine counts of various populations at different travel times to safety. In addition, the software allows researchers considerable latitude in the construction of the land-cover cost inverse layer. A base land-cover layer can be augmented with any number of additional layers, such as roads, buildings, water, and trails, when creating the cost-inverse raster. This flexibility lets researchers easily modify the composite land cover to examine different

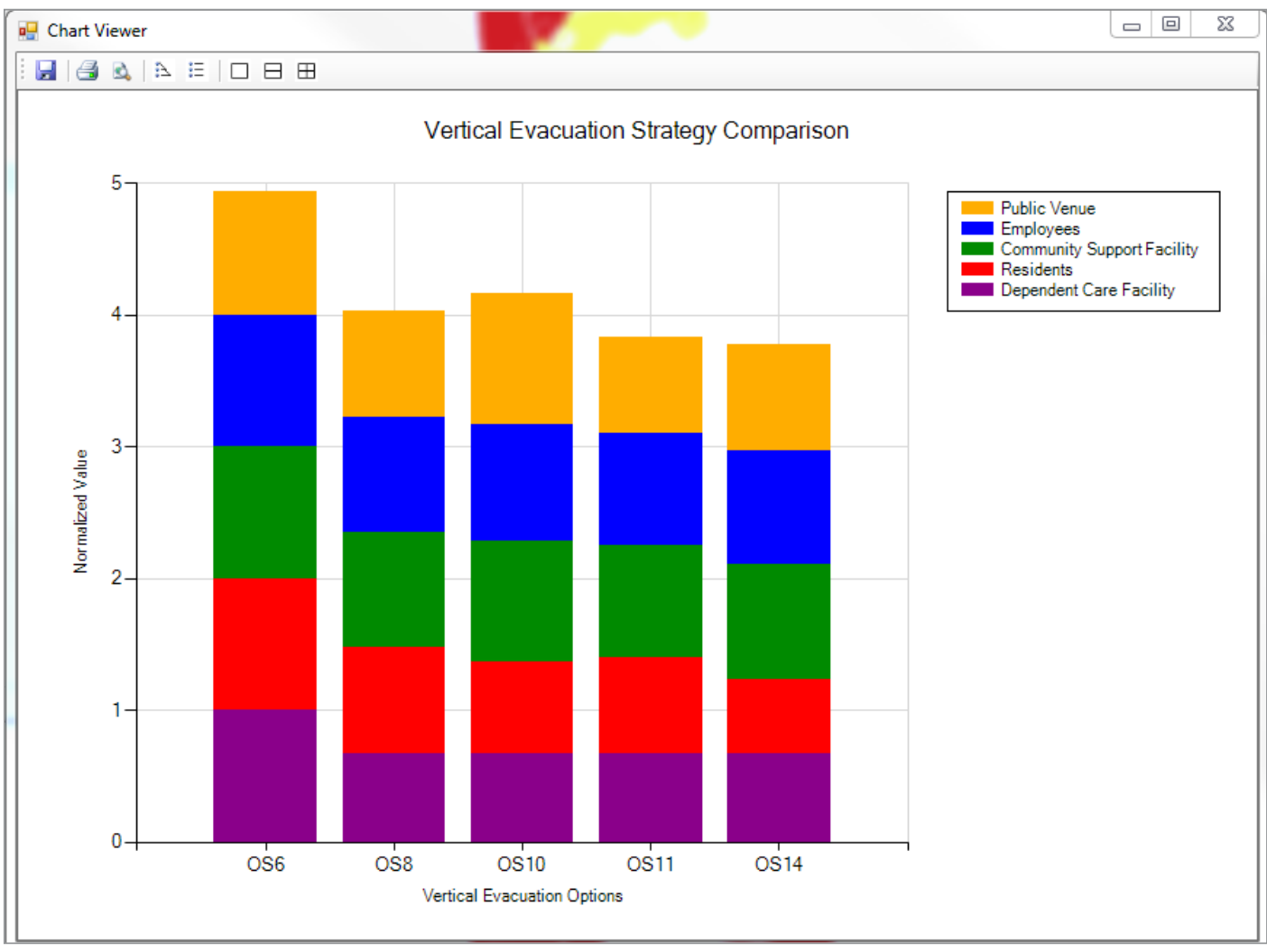

Figure 26. Screenshot of Vertical Evacuation Strategy Comparison chart results in the Pedestrian Evacuation Analyst. 
scenarios such as evacuation potential with and without bridges in place.

The initial release of the software features a simplified design geared toward a researcher analyzing a single study area, and the size of this study area is limited by what ArcGIS can process. The ability to streamline the processing of multiple study areas would help in cases where a large study area needs to be broken up into smaller areas for processing. Also, the software currently has limited ability to compare multiple scenarios, a feature which would assist the researcher performing a regional study.

Given these limitations, future improvements to the tool could include (1) comparison of multiple scenarios, (2) aggregation of multiple scenario results into a larger time map for population processing, and (3) the ability to automate the processing of multiple study areas. In addition, new features to enhance decision support could include a site suggestion option that takes input and results layers and determines possible sites for vertical-evacuation options. It is hoped that this tool will assist hazard research both within the U.S. Geological Survey (USGS) and in the wider research community, providing valuable information on strategies for minimizing loss of life in catastrophic events.

\section{Acknowledgments}

This study was supported by the USGS Land Change Science Program. We thank Mara Tongue and Jamie Ratliff of the USGS, Amy MacPherson at the Geophysical Institute at the University of Alaska Fairbanks, and a group of researchers led by Dr. Tim Frazier at the University of Idaho for their insightful reviews of earlier versions of the report.

\section{References Cited}

Esri, 2014, ArcGIS Help 10.1-Understanding path distance analysis: Esri ArcGIS Resources Web site, accessed February 24, 2014, at http://resources.arcgis.com/en/help/ main/10.1/index.html\#/Understanding_path_distance_ analysis/009z00000022000000/.

Federal Geographic Data Committee, 2014, Geospatial Metadata: Federal Geographic Data Committee Web site, accessed February 21, 2014, at http://www.fgdc.gov/ metadata.

Intergovernmental Oceanographic Commission, 2002, Progress report of ad hoc working group on international tsunami signs and symbols: United Nations Educational, Scientific and Cultural Organization, IOC/ITSU-ON-2003/9, 7 p.
MarathonGuide.com, 2011, Boston marathon-race results, April 19, 2010: MarathonGuide.com Web site, accessed March 8, 2011, at http:/www.marathonguide.com/results/ browse.cfm?MIDD=15100419.

Soule, R.G., and Goldman, R.F., 1972, Terrain coefficients for energy cost prediction: Journal of Applied Physiology, v. 32, no. 5, p. 706-708.

Federal Highway Administration, 2009, Manual on uniform traffic control devices for streets and highways, 2009 edition: Federal Highway Administration,816 p.

Walsh, T., Caruthers, C., Heinitz, A., Myers, E., III, Baptista, A., Erdakos, G., Kamphaus, R., 2000, Tsunami hazard map of the southern Washington coast-modeled tsunami inundation from a Cascadia subduction zone earthquake: Washington Department of Natural Resources Division of Geology and Earth Resources Geologic Map GM-49.

Wood, N., and Schmidtlein, M., 2012, Anisotropic path modeling to assess pedestrian-evacuation potential from Cascadia-related tsunamis in the US Pacific Northwest: Natural Hazards, v. 62, no. 2, p. 275-300, doi 10.1007/ s11069-011-9994-2.

Wood, N., and Schmidtlein, M., 2013, Community variations in population exposure to near-field tsunami hazards as a function of pedestrian travel time to safety: Natural Hazards, v. 65 , no. 3 , p. 1603-1628.

\section{Appendix A. Line-Data Representation}

During land-cover preprocessing in the Pedestrian Evacuation Analyst, the user can input line data such as roads as either line shapefiles or buffer the roads to some reasonable width and enter them as polygons. If entering line data in raster format, note that different geographic information system (GIS) software packages may represent line data in different formats when converted to raster data. Figure 27 shows the same curved line vector converted to raster in different GIS applications. The raster pixel representation on the left is "corner to corner" format, and on the right, the pixel representation has more overlap in a "staircase" format.

The documentation on Esri's path-distance tool (Esri, 2014) states that the algorithm considers all eight directions (sides and corners) out of a cell when choosing a path to safety. For thin, corner-to-corner barriers, the cell corners on each side of the fence are touching and provide a path for the algorithm to cross. In this case, the curved fence line does not serve as a barrier to travel. When curved lines are represented in a thicker, staircase pixel format, the path distance algorithm does not see a path through the fence. Figure 28 shows time maps generated around a fenced-in ball field for each type of line representation. With the corner-to-corner arrangement 

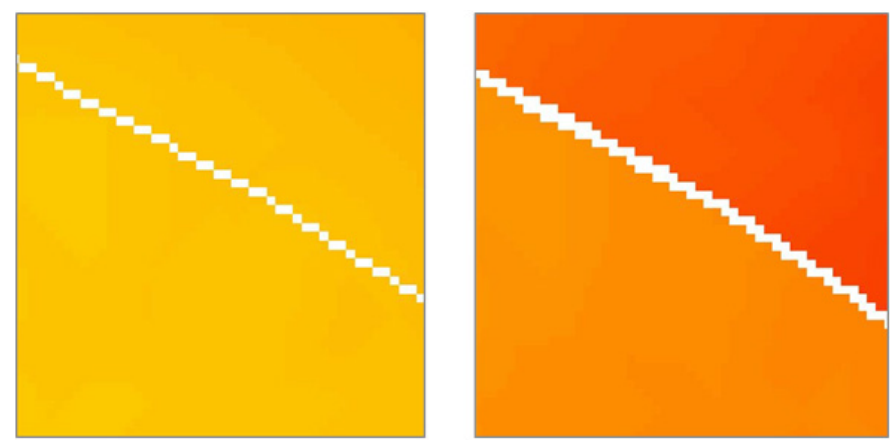

Figure 27. Images of line representation in corner-to-corner (left) and staircase (right) formats.

(fig. 28, left), the curved fence is transparent to the path distance algorithm.

ArcGIS converts line-vector data into raster data with the staircase pixel arrangement and produces the expected results in the time map. Care should be taken when using land cover layers generated in other GIS applications. When in doubt, enter any line-type ancillary land-cover data in vector format and let ArcGIS handle the conversion to raster in the Pedestrian Evacuation Analyst software.

\section{Appendix B. Tool-Folder Structure}

When a portfolio is created in the Portfolio Management window of Pedestrian Evacuation Analyst, the software creates an initial set of folders in the results and workspace folders selected by the user. As each processing step is run, the tool creates more folders to hold the processed files. Preprocessing, path distance, and evacuation-time surface steps store output in the workspace file path, whereas time-map and population processing steps store output in the results file path. The population processing also creates a folder in the workspace file path to hold the preprocessed population files. Under the workspace file path, the ETS folder name is for the evacuation-time surface and the LULC folder name is for land-cover cost inverse. Under the results file path, the ETM folder name is for evacuation time map and VE is for vertical evacuation, and the xml folder contains XML (extensible markup language) text files with records of the parameters used for each processing step.

When processing occurs on steps that have a travel speed associated with them, travel-speed folders are created within that processing step's folder to differentiate between the output at different speeds. For population results, the table data (.csv files) and the population-type shapefiles with travel times appended (identity files) are stored in folders by travel speed,
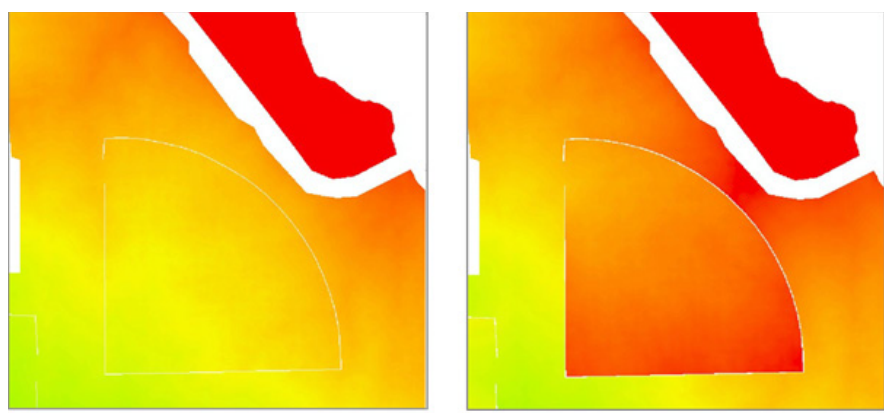

Figure 28. Images of algorithm results with corner-to-corner (left) and staircase (right) line representations.

and if VE layers were included in the population processing, the folder name is appended with " VE". For example, population processing for Taholah, Washington, at a slow walk with no VE structures specified generates a SlwWlk folder under the Population folder. Running population processing again for Taholah at a slow walk and adding VE structures generates a SlwWlk_VE folder. The contents of these two folders may be identical; the distinction is made to keep each processing thread intact and to logically identify folders for the graphing module. The SlwWlk folder holds the stand-alone processing results, whereas the SlwWlk_VE folder holds the baseline processing results for a slow-walk travel speed. The corresponding VE processing results are located in the VE folder for the portfolio in the results file path and are again grouped by travel speed.

The results of VE processing, both time maps and population tables and files, are located in the results file path (results folder $>$ portfolio name $>\mathrm{VE}>$ travel speed). Within the travel speed folder, each VE has its own subfolder holding the time map for that VE, the .csv files, and the population identity files. The workspace folders generated for each VE structure are deleted after processing is complete to conserve disk space.

\section{Appendix C. File-Naming and Storage Convention}

The Pedestrian Evacuation Analyst creates numerous files in the workspace, results, and scratch areas for use in processing and for examination by the user. The names and locations for some of these files can be selected by the user in the tool windows, but if default names and locations are chosen, they will follow a naming convention that is described in table 5. 
Table 5. File-naming and storage convention in the Pedestrian Evacuation Analyst.

[A word in parentheses represents a placeholder for an actual folder or file name. For example, folders created for the Taholah_WA portfolio name will have Taholah_WA where "(portfolio name)" appears in the table file path. DEM, digital elevation model; LULC, land use/land cover; ETS, evacuation-time surface; ETM, evacuation-time map; VE, vertical evacuation; MSZ, merge safe zone]

\begin{tabular}{|c|c|c|}
\hline Tool & Output name & File path \\
\hline DEM preprocess & $\begin{array}{l}\text { dem_r } \\
\text { study_v.shp } \\
\text { study_r }\end{array}$ & $\begin{array}{l}(\text { workspace })>(\text { portfolio name })>D E M \\
(\text { workspace })>(\text { portfolio name })>\text { StudyArea }\end{array}$ \\
\hline Land-cover preprocess & tci_r & (workspace) $>$ (portfolio name $)>$ LULC \\
\hline Hazard-zone preprocess & $\begin{array}{l}\text { preliminary_safe_zone.shp } \\
\text { tlcps_r }\end{array}$ & $\begin{array}{l}(\text { workspace })>(\text { portfolio name })>\text { HazardZone } \\
(\text { workspace })>(\text { portfolio name })>\text { PathDistance }\end{array}$ \\
\hline Evacuation surface & tets_r & (workspace) $>($ portfolio name $)>$ ETS $>($ travel speed $)$ \\
\hline Time map & $\begin{array}{l}\text { tetm_r } \\
\text { tetm_r_fil }{ }^{1} \\
\text { TETM_V.shp } \\
\text { TETM_V_fill.shp }\end{array}$ & (results) $>$ (portfolio name $)>$ ETM $>$ (travel speed $)$ \\
\hline Safe-zone merge & MSZ_TSZ_V.shp & (workspace $)>($ portfolio name $)>M S Z$ \\
\hline Population & $\begin{array}{l}\text { (popname).csv }{ }^{4} \\
\text { (popname)_expand.csv } \\
\text { (popname)_identity.shp }\end{array}$ & $\begin{array}{l}(\text { results })>(\text { portfolio name })>\text { Population }>(\text { travel speed }) \\
(\text { results })>(\text { portfolio name })>\text { Population }>(\text { travel speed }) \\
(\text { results })>(\text { portfolio name })>\text { Population }>(\text { travel speed }) \mathrm{VE}^{5}\end{array}$ \\
\hline
\end{tabular}

\footnotetext{
${ }^{1}$ The versions of the time map with "fil" or "fill" appended have building footprints filled in with the minimum value of the time map around the perimeter of the footprint. These versions are used with the population-point files to extract travel time.

${ }^{2}$ The "ve_id" in the names and for the folder will be the VE structure name from the input file or a basic numbering scheme chosen by the tool.

${ }^{3}$ The "(ve_id)_structure.shp" file contains the VE polygon.

${ }^{4}$ The "popname" in the names will be the population type, such as residents and employees.

${ }^{5}$ For baseline results associated with VE processing, the foler will have “_VE” appended.
} 
This page is left intentionally blank 
Menlo Park Publishing Service Center, California

Manuscript approval date July 15, 2014

Edited by James W. Hendley II

Design and layout by Vivian Nguyen 
